\title{
SuELen MONTAGNeR
}

Estudo comparativo da sobrevida de pacientes com melanoma ungueal tratados com cirurgia funcional ou amputação distal

Dissertação apresentada à Faculdade de Medicina da Universidade de São Paulo para obtenção do título de Mestre em Ciências

Programa de Dermatologia

Orientador: Prof. Dr. Walter Belda Junior

São Paulo

2016 


\section{SuELen MONTAGNeR}

Estudo comparativo da sobrevida de pacientes com melanoma ungueal tratados com cirurgia funcional ou amputação distal

Dissertação apresentada à Faculdade de Medicina da Universidade de São Paulo para obtenção do título de Mestre em Ciências

Programa de Dermatologia

Orientador: Prof. Dr. Walter Belda Junior

São Paulo

2016 
Dados Internacionais de Catalogação na Publicação (CIP)

Preparada pela Biblioteca da Faculdade de Medicina da Universidade de São Paulo (C)reprodução autorizada pelo autor

Montagner, Suelen

Estudo comparativo da sobrevida de pacientes com melanoma ungueal tratados com cirurgia funcional ou amputação distal/ Suelen Montagner - São Paulo, 2016.

Dissertação (mestrado)—Faculdade de Medicina da Universidade de São Paulo. Programa de Dermatologia.

Orientador: Walter Belda Junior.

Descritores: 1. Melanoma 2. Doenças da unha 3. Cirurgia 4. Análise de sobrevida 5. Terapia 
A todos que incentivam minha caminhada profissional.

Em especial:

Aos meus pais Célia e Ailton, por sempre acreditarem em mim e serem meu alicerce.

À minha querida irmã, pelo apoio constante.

Ao meu esposo Emerson, companheiro sempre presente.

E ao meu pequeno Giovanni, a quem um dia eu possa inspirar. 


\section{AGRADECIMENTOS}

A Deus, por abrir meus caminhos e estar sempre ao meu lado.

Ao Prof. Dr. Walter Belda Junior, profissional admirável, agradeço pela disponibilidade, cuidadosa orientação e pelo aprendizado.

Ao Prof. Dr. Nilton di Chiacchio, generoso dermatologista, que me despertou para o estudo das unhas e tanto me incentiva nessa caminhada. Sem ele, esse trabalho não teria sido possível.

Ao Prof. Dr. Francisco Aparecido Belfort, pela generosidade em contribuir com parte da casuística.

Aos meus amigos e familiares, pelo apoio e incentivo. 


\section{NORMATIZAÇÃO ADOTADA}

Esta dissertação está de acordo com as seguintes normas, em vigor no momento desta publicação:

Referências: adaptado de International Committee of Medical Journals Editors (Vancouver).

Universidade de São Paulo. Faculdade de Medicina. Divisão de Biblioteca e Documentação. Guia de apresentação de dissertações, teses e monografias. Elaborado por Anneliese Carneiro da Cunha, Maria Julia de A. L. Freddi, Maria F. Crestana, Marinalva de Souza Aragão, Suely Campos Cardoso, Valéria Vilhena. 3a ed. São Paulo: Divisão de Biblioteca e Documentação; 2011.

Abreviaturas dos títulos dos periódicos de acordo com List of Journals Indexed in Index Medicus. 


\section{SUMÁRIO}

Lista de abreviaturas, símbolos e siglas

Lista de tabelas

Lista de figuras

Lista de quadros

Resumo

Abstract

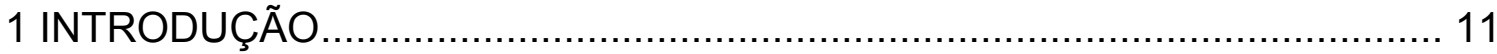

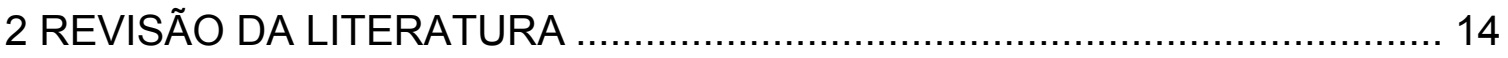

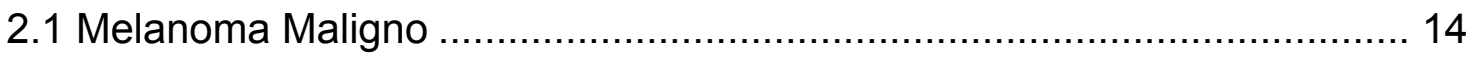

2.1.1 Melanoma ungueal........................................................ 15

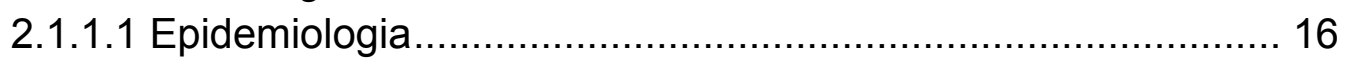

2.1.1.2 Etiologia .................................................................... 16

2.1.1.3 Manifestação clínica................................................... 16

2.1.1.4 Diagnóstico ................................................................ 17

2.1.1.5 Histopatologia ....................................................... 22

2.1.1.6 Tratamento ................................................................. 23

2.1.1.7 Amputação ............................................................... 24

2.1.1.8 Cirurgia funcional ..................................................... 26

2.1.1.9 Prognóstico ..................................................................... 28

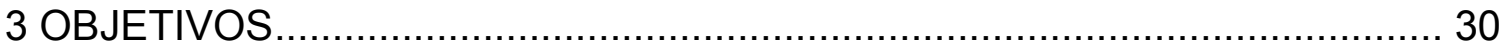

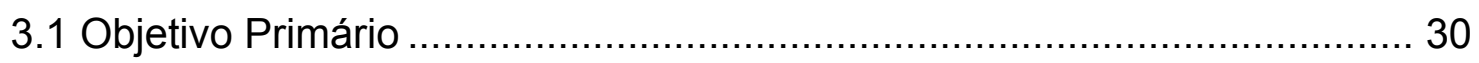

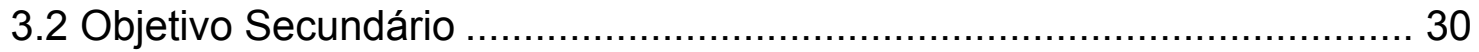

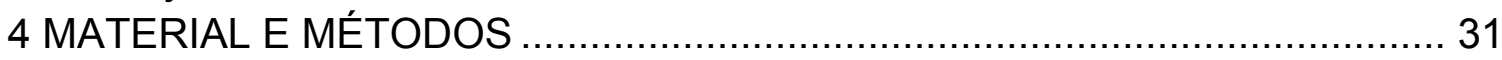

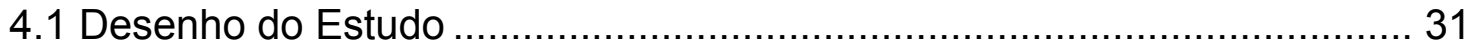

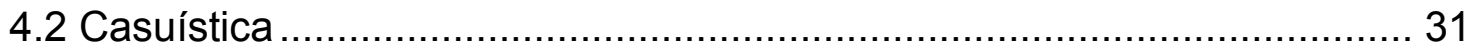

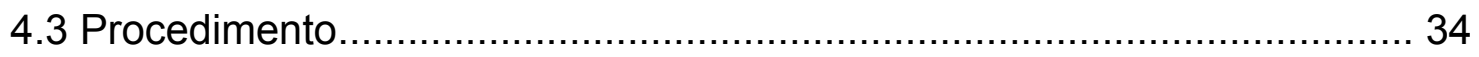

4.4 Análise Estatística ........................................................................ 34

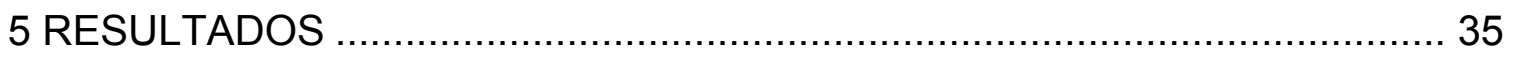

5.1 Análise Descritiva - Caracterização da Amostra................................... 35

6 DISCUSSÃO ............................................................................... 41

6.1 Aspectos Gerais das Características Clínicas e Sobrevida..................... 41

7 CONCLUSÃO .................................................................................... 45

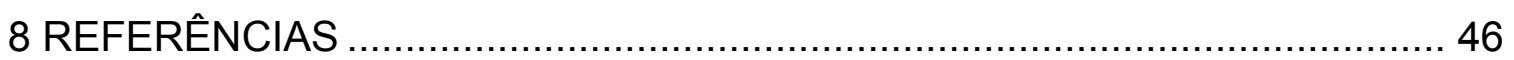




\section{LISTA DE ABREVIATURAS, SÍMBOLOS E SIGLAS}

AJCC American Joint Committee on Cancer

BRAF Oncogene B-Raf

LDH Desidrogenase láctica

MU Melanoma ungueal

MLA Melanoma lentiginoso acral

TNM Classificação de tumores malignos ( $\mathrm{T}$ : descreve o tamanho do tumor, $\mathrm{N}$ : descreve o acometimento linfonodal, $\mathrm{M}$ : descreve presença de metástase a distância) 


\section{LISTA DE TABELAS}

Tabela 1-Características clínicas e histopatológicas dos casos, estratificados por cirurgia funcional e amputação

Tabela 2 - Medidas de posição e dispersão da idade e do tempo de seguimento dos pacientes

Tabela 3 - Resultados da regressão simples de Cox 37

Tabela 4-Casos in situ da literatura tratados com cirurgias conservadoras, e tempo em que ocorreram as respectivas recidivas 


\section{LISTA DE FIGURAS}

Figura 1 - Representação da anatomia ungueal, secção longitudinal do dedo.

Figura 2 - Técnica de biópsia por excisão tangencial ................................... 22

Figura 3 - Evolução do nível de amputação ................................................ 25

Figura 4 - Técnica conservadora, com preservação da falange ....................... 28

Figura 5 - Representação gráfica da distribuição dos pacientes com melanoma ungueal e cutâneo na fase I de estadiamento TNM ....... 29

Figura 6 - Curva de sobrevivência global ................................................ 38

Figura 7 - Correlação entre sobrevida livre de doença e a faixa etária $(<=56$ anos ou $>56$ anos $)$...................................................... 38

Figura 8 - Correlação entre sobrevida livre de doença e o gênero................... 39

Figura 9 - Correlação entre sobrevida livre de doença e a localização, seja no hálux ou nos demais dedos agrupados como "não hálux"

Figura 10 - Correlação entre sobrevida livre de doença e o seguimento dos pacientes de acordo com a técnica cirúrgica adotada para tratamento do melanoma ungueal (amputação versus cirurgia

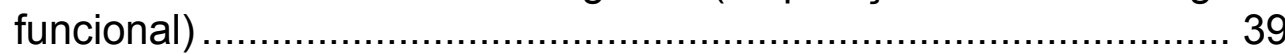

Figura 11 - Correlação entre sobrevida livre de doença e espessura tumoral ..40

Figura 12 - Correlação entre sobrevida livre de doença e presença de ulceração à histopatologia

Figura 13 - Correlação entre sobrevida livre de doença e presença de mitose à histopatologia $(p=0,0511)$. 


\section{LISTA DE QUADROS}

Quadro 1-Regra do ABCDEF, para avaliação de possível melanoma ungueal ....................................................................... 18

Quadro 2 - Casos de melanoma ungueal estudados entre 2003 e 2013 ......32

Quadro 3 - Características dos pacientes e respectivos desfechos ...............36

Quadro 4-Distribuição em frequência e porcentagem das variáveis categóricas …….................................................................. 


\section{RESUMO}

Montagner S. Estudo comparativo da sobrevida de pacientes com melanoma ungueal tratados com cirurgia funcional ou amputação distal [dissertação]. São Paulo: Faculdade de Medicina, Universidade de São Paulo; 2016.

O melanoma ungueal compreende cerca de 0,7 a $3,5 \%$ de todos os tipos de melanoma. Devido à dificuldade no diagnóstico precoce, seu prognóstico é reservado quando comparado às demais formas de melanoma. A amputação falangeana se propõe a extirpar o tumor respeitando os princípios da cirurgia oncológica, apesar de acarretar déficit funcional e estigmas sociais, além de não prolongar a sobrevida, particularmente nos casos iniciais. Abordagens mais conservadoras, como a cirurgia funcional, são utilizadas em casos in situ ou microinvasivos, que consistem na remoção de todo o complexo ungueal e preservação da falange distal, com bom resultado cosmético e funcional. Este estudo retrospectivo propõe comparar a sobrevida de pacientes tratados em cada uma das técnicas mencionadas, a partir de dados coletados de prontuários. Não houve alteração da sobrevida dos pacientes com melanoma ungueal tratados com amputação ou cirurgia funcional.

Descritores: Melanoma; Doenças da unha; Cirurgia; Análise de sobrevida; Terapia. 


\begin{abstract}
Montagner S. Comparative study of nail melanoma patients' survival following treatment with functional surgery or distal amputation [dissertation]. São Paulo: University of São Paulo Medical School; 2016.

Nail melanoma represents about $0.7-3.5 \%$ of all types of melanoma. Because its early diagnosis is difficult, the prognosis is conservative when compared with other forms of melanoma. The phalangeal amputation is intended to excise the tumor according to the cancer surgery principles, despite resulting in functional deficit and social stigmata and failing to extend survival, especially in early cases. More conservative approaches, such as functional surgery, have been used in 'in situ' or microinvasive cases, which consist of removing the entire nail complex and preserving the distal phalange, provide good aesthetic and functional results. This retrospective study proposes to compare the survival of patients treated with each of these techniques by reviewing the data collected from their medical records. No changes were observed in the survival of nail melanoma patients treated with amputation or functional surgery.
\end{abstract}

Key words: Melanoma; Nail diseases; Surgery; Survival analysis; Therapy. 


\section{INTRODUÇÃO}

O melanoma ungueal (MU), ou subungueal, compreende cerca de $0,7 \%$ a $3,5 \%$ de todos os tipos de melanoma, sendo uma variante do melanoma lentiginoso acral (MLA). ${ }^{1,2}$ Embora sua incidência em número absoluto seja semelhante entre os diferentes grupos étnicos, representa $1,5 \%$ a $2 \%$ dos melanomas em caucasianos, comparado a mais de $20 \%$ nos indivíduos negros e orientais. ${ }^{1,3,4}$ A população entre 50 e 70 anos é a mais acometida, mas também pode ser observado em crianças. ${ }^{5}$

Origina-se na matriz ungueal, podendo envolver a região periungueal ou ainda outros componentes do aparelho ungueal, como o leito e o hiponíquio. ${ }^{2,6}$ Atinge com maior frequência o polegar e o hálux, seguidos pelo indicador $\mathrm{e}$ dedo médio. Sua localização é menos comum nos pododáctilos que nos quirodáctilos, ao contrário do melanoma acral, que é mais frequente nos pés do que nas mãos. ${ }^{7,8}$

No estágio inicial, o MU geralmente se apresenta como uma pigmentação na placa ungueal, conhecida por melanoníquia estriada. ${ }^{9-11}$ Muitas vezes, os pacientes notam a hiperpigmentação após um trauma local. No entanto, devido à dificuldade de diagnóstico precoce, possui prognóstico reservado e baixa sobrevida quando comparado às demais formas de melanoma. A interferência da placa ungueal, alterando a reflexão da luz, e o aspecto amelanótico da lesão (15-65\% dos casos) são fatores que contribuem para a detecção tardia. ${ }^{12-14}$

Para abordar tamanha agressividade neoplásica, a técnica amplamente difundida como primeira escolha terapêutica se mostra proporcionalmente agressiva. Trata-se da amputação falangeana ${ }^{15,16}$, cirurgia que se propõe a extirpar o tumor, respeitando os princípios da cirurgia oncológica, apesar de acarretar déficit funcional e estigmas sociais, além de não prolongar a sobrevida, particularmente nos casos iniciais. ${ }^{17}$ 
Esse procedimento provém da evolução de técnicas ainda mais radicais, sendo que a comparação dos diferentes níveis de amputação não demonstrou qualquer vantagem de sua realização no metacarpo sobre as articulações metacarpo-falangeana, interfalangeanas proximal ou distal. ${ }^{18-23}$

Contudo, há mais de 30 anos, o manejo do MU vem sendo questionado e redefinido. Abordagens mais conservadoras são propostas para casos diagnosticados precocemente, com resultado favorável. ${ }^{14,24} \mathrm{O}$ momento do diagnóstico, de acordo com o nível de invasão tumoral, passa a determinar o tipo de cirurgia a ser adotada. ${ }^{2}$ Essa evolução preserva a funcionalidade, sem comprometer os princípios terapêuticos. ${ }^{25}$

Em 2002, Clarkson e colaboradores ${ }^{26}$ excisaram dois melanomas in situ da mesma unha, por meio da remoção de todo o complexo ungueal com preservação da falange distal, seguido de reconstrução por retalho. ${ }^{26}$ Esse tipo conservador de cirurgia vem sendo indicado no tratamento do $\mathrm{MU}$ in situ com bom resultado cosmético e funcional, sem alterar o prognóstico. Outros autores também demonstraram o sucesso terapêutico, não apresentando recidiva. ${ }^{1,26-30}$ Até 2011, dos 29 casos de melanoma in situ tratados com a cirurgia conservadora na literatura, 3 foram submetidos à amputação devido margem positiva e outros 2 recidivaram tempo após a cirurgia. ${ }^{1}$

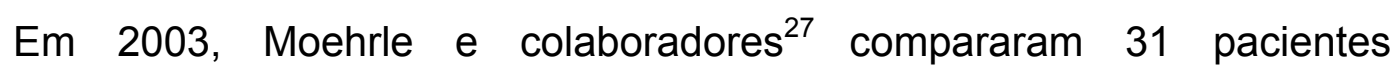
submetidos à amputação com 31 pacientes tratados com a remoção do complexo ungueal e preservação da falange distal. A sobrevida em 5 anos encontrada no primeiro grupo foi de $66,5 \%$ contra $91,7 \%$ no segundo.

A despeito da divulgação da técnica por meio de alguns trabalhos com resultados otimistas, muitos cirurgiões mantêm a cirurgia radical como primeira escolha, devido à escassez de estudos comparativos em número e tempo de seguimento clínico.

Nesse contexto, propusemos a avaliação comparativa da sobrevida de pacientes com MU tratados com amputação ou cirurgia funcional. 


\section{REVISÃO DA LITERATURA}

\subsection{Melanoma Maligno}

Atualmente, 132 mil casos de melanoma são diagnosticados no mundo, a cada ano. ${ }^{31}$ No Brasil, representa $4 \%$ das neoplasias malignas cutâneas, porém é a mais grave delas. Segundo o Instituto Nacional do Câncer, estimase 5.670 novos casos para o ano de 2016, dos quais, 3 mil serão homens e 2.670, mulheres. ${ }^{32}$

O melanoma maligno cutâneo é responsável por $78 \%$ dos óbitos por câncer de pele, sendo a forma mais letal dentre eles $^{33}$, e a exposição à radiação ultravioleta é um importante fator de risco. ${ }^{34}$ Existem quatro subtipos histológicos. O extensivo superficial, mais comum, corresponde a $70 \%$ dos casos e é mais frequente no tronco. O melanoma nodular representa $15 \%$ dos casos e apresenta apenas a fase de crescimento vertical. O lentigo melanoma é responsável por $13 \%$ dos melanomas e se correlaciona com a exposição solar prolongada em indivíduos de pele clara e com mais idade. ${ }^{35}$

O melanoma lentiginoso acral é o tipo mais raro, somando $2 \%$ a $3 \%$ de todos os melanomas. Acomete predominantemente o leito ungueal, as palmas e as plantas. ${ }^{35,36}$ O termo "acral" implica sua localização anatômica e "lentiginoso" se refere ao padrão histopatológico, de crescimento radial. ${ }^{8}$

Os melanomas acrais são mais frequentes nos pés (73\%) do que nas mãos $(27 \%) .^{8}$ Geneticamente, se distinguem dos cutâneos. Amplificações de genes foram demonstradas em $100 \%$ dos 15 casos de melanomas acrais estudados, enquanto apenas 2 dos 15 extensivos superficiais a apresentavam. As regiões mais frequentemente amplificadas foram 11q13 (47\%) e 22q11-13 (40\%). Essas amplificações foram detectadas na fase in situ e também são encontradas em melanócitos intraepidérmicos isolados de pele normal adjacente. ${ }^{37} \mathrm{O}$ melanoma lentiginoso acral apresenta uma menor taxa de mutação do oncogene BRAF (15\%) quando comparado ao melanoma no tronco $(53 \%){ }^{38-43}$ As mutações no BRAF são relativamente frequentes nos melanomas metastáticos, sendo que o 
BRAF-V600E é observado em $50 \%$ dos casos. Esse dado pode ter relevância no tratamento dos melanomas metastáticos, que conta com os recentes avanços na imunoterapia. ${ }^{44}$

\subsubsection{Melanoma ungueal}

O MU, por sua vez, é um raro subtipo de melanoma lentiginoso acral, que se origina na matriz e pode envolver a região periungueal ou ainda outros componentes do aparelho ungueal, como o leito e o hiponíquio, que podem ser observados na Figura $1^{2,6}$ Compreende de $0,7 \%$ a $3,5 \%$ de todos os melanomas cutâneos. ${ }^{1,2}$

Foi descrito pela primeira vez por Boyer, em 1834, e posteriormente nomeado por Hutchinson, em 1886, como melanotic whitlow (panarício melanótico), por seu curso maligno e semelhança com o panarício, exceto pela pigmentação. ${ }^{45,46}$

Devido à raridade, dados referentes à epidemiologia, à etiologia, ao comportamento clínico em longo prazo e ao tratamento ideal não estão bem estabelecidos. ${ }^{8,47}$

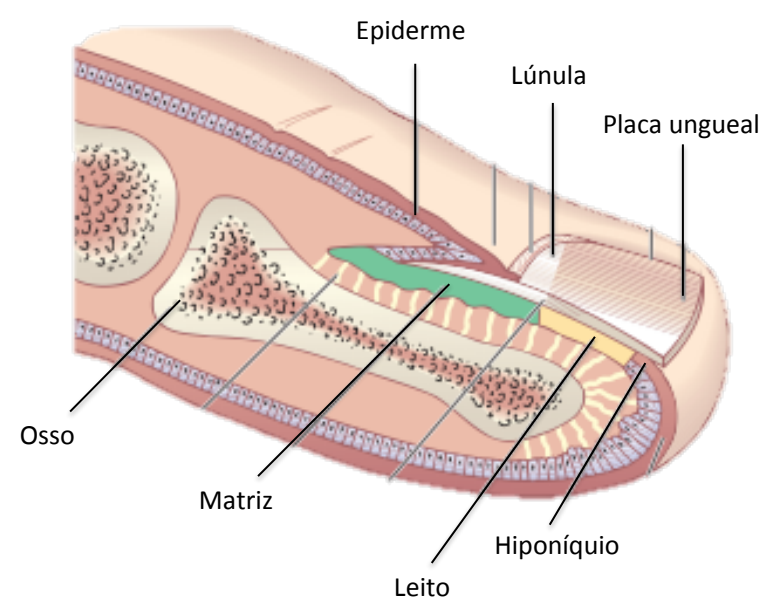

FONTE (adaptado de): Berker DAR, Baran R. Disorder of Nails. In: Burns T, Breathnach S, Cox N, Griffiths C, editors. Rook's Textbook of Dermatology. Oxford: Blackwell Publishers; 2010. Chap.65, p 1-57.

Figura 1 - Representação da anatomia ungueal, secção longitudinal do dedo 


\subsubsection{Epidemiologia}

Embora sua incidência em número absoluto seja semelhante entre os diferentes grupos étnicos, representa $1,5 \%$ a $2,0 \%$ dos melanomas em caucasianos, comparado a mais de $20 \%$ nos indivíduos negros e orientais. ${ }^{1,3,4}$ A população entre 50 e 70 anos é a mais acometida, mas também pode ser observado em crianças. ${ }^{5,49-51}$

Preferencialmente, acomete o polegar e o hálux, seguidos pelos dedos indicador e médio; a localização ungueal acomete mais os dedos das mãos (52\% a $76 \%)$ que os dos pés ${ }^{7,8,52-56}$

\subsubsection{Etiologia}

Ao contrário do melanoma cutâneo, parece pouco provável que radiação significante penetre na placa ungueal. ${ }^{8,57} \mathrm{~A}$ unha é formada por uma camada compacta de queratina, que age como um escudo à luminosidade. ${ }^{58}$ Além disso, a unha do hálux é frequentemente afetada, mesmo nos indivíduos que não tenham o hábito de andar descalços. ${ }^{2}$

Muitas vezes, os pacientes referem o surgimento da lesão após trauma local, que pode ser agudo ou crônico, e parece desempenhar um importante papel na etiologia do MU. Tal trauma pode ser interpretado como: uma simples coincidência, uma maior atenção para a área escura sob a unha, desencadeante do sangramento de uma lesão subclínica ou, ainda, indutor de mutação melanocítica. ${ }^{56}$

\subsubsection{Manifestação clínica}

No estágio inicial, o MU geralmente se apresenta como uma faixa de 
coloração marrom ou preta na placa ungueal, conhecida por melanoníquia estriada ou longitudinal. ${ }^{9-11}$ Essa pigmentação pode ser melanina ou pigmentos exógenos, e o reconhecimento da melanina geralmente necessita de confirmação histopatológica. A melanoníquia é clinicamente significante, pois dois terços dos melanomas ungueais se manifestam dessa forma, porém é uma lesão inespecífica. ${ }^{59,60}$ São sinais preditivos de melanoma o envolvimento das dobras ungueais e hiponíquio, a distrofia da placa, o estreitamento distal da lesão e o centro escuro com borramento das laterias. ${ }^{9-11}$

O pigmento castanho enegrecido do leito ungueal, matriz ou placa, pode se estender para as pregas ungueais proximal ou laterais, descrito como sinal de Hutchinson. ${ }^{61,62}$ Embora seja uma característica clínica valiosa, não é um sinal patognomônico da doença $a^{63}$, trata-se de um sinal clínico da fase de crescimento radial do tumor. ${ }^{64,65-67}$

Outros sinais clínicos sugestivos de MU seriam distrofia na placa com lesão exsudativa ou sangrante, semelhante à unha encravada, porém em localização ou idade atípicas. ${ }^{2} \mathrm{O}$ melanoma amelanótico também pode se manifestar com distrofia da placa ungueal, muitas vezes parcialmente destruída por uma lesão vegetante, eritematosa e hemorrágica. ${ }^{11}$ Alterações distróficas geralmente ocorrem na fase de crescimento vertical do tumor. ${ }^{63}$

O diagnóstico diferencial para MU amelanótico inclui granuloma piogênico, paroníquia crônica, tumor vascular, ${ }^{48}$ carcinoma espinocelular e exostose subungueal. ${ }^{68}$ Já as lesões pigmentadas que se apresentam como melanoníquia estriada, podem ser causadas por nevo da matriz ungueal, hiperplasia melanocítica benigna (lentigo) e outras desordens inflamatórias, traumáticas ou iatrogênicas que induzem a ativação dos melanócitos da matriz. $^{69}$

\subsubsection{Diagnóstico}

O diagnóstico precoce do MU ainda é um desafio na Dermatologia. Sua apresentação clínica pode ser sugestiva, mas não é patognomônica, uma vez que a melanoníquia estriada também pode ser observada em condições 
benignas resultantes da ativação melanocítica, como hipermelanoses, ou da proliferação melanocítica, como lentigo ou nevo. ${ }^{69-73}$

De forma análoga ao $A B C D$ do melanoma, a regra do $A B C D E F$ (Quadro 1) é utilizada como um método mnemônico para facilitar a identificação clínica de uma lesão ungueal suspeita de melanoma. ${ }^{49}$

Quadro 1 - Regra do ABCDEF, para avaliação de possível melanoma ungueal

\begin{tabular}{|c|c|}
\hline LETRA & REGRA \\
\hline $\begin{array}{c}\mathbf{A} \\
(\mathrm{Age})\end{array}$ & $\begin{array}{l}\text { Varia de } 20 \text { a } 90 \text { anos (pico entre a quinta e sétima décadas de vida); Etnias: } \\
\text { afro-americanos, asiáticos e nativo-americanos }\end{array}$ \\
\hline $\begin{array}{c}\text { B } \\
\text { (Band) }\end{array}$ & Faixa pigmentada (marrom ou preta), espessura $\geq 3 \mathrm{~mm}$, borda irregular \\
\hline $\begin{array}{c}\text { C } \\
\text { (Change) }\end{array}$ & Aumento abrupto no tamanho ou alteração da morfologia \\
\hline $\begin{array}{c}\text { D } \\
\text { (Digit) }\end{array}$ & Acometimento do dedo (polegar, hálux e indicador), único dedo x múltiplos \\
\hline $\begin{array}{c}\mathbf{E} \\
\text { (Extension) }\end{array}$ & Envolvimento das bordas ungueais \\
\hline $\begin{array}{l}\mathbf{F} \\
\text { (Family) }\end{array}$ & História familiar ou pessoal de melanoma ou síndrome do nevo displásico \\
\hline
\end{tabular}

FONTE (adaptado de): Levit EK, Kagen MH, Scher RK, et al. The ABC rule for clinical detection of subungual melanoma. J Am Acad Dermatol. 2000;42:269-74.

A letra "A" se refere à idade (age, em inglês) de apresentação e à etnia. A idade mais acometida varia de 20 a 90 anos $^{6,67,74-77}$, com pico entre a quinta e sétima décadas de vida. $3,74,75,78-80$

Em relação à etnia, observa-se que o MU é mais frequente em pessoas de alto fototipo, representando de $15 \%$ a $20 \%$ de todos os melanomas em afroamericanos $^{70}, \quad 10 \%$ a $31 \%$ em asiáticos ${ }^{3,67,70,74,81}$ e $33 \%$ em nativoamericanos. $^{82}$

A letra "B" se refere à apresentação clínica mais típica, que é uma faixa (band, em inglês) marrom ou preta na unha. ${ }^{82,83}$ Durante o exame, deve-se atentar para os tons de pigmento, largura e limites da borda. A presença de tonalidades variadas, ${ }^{81}$ largura acima de $3 \mathrm{~mm}^{84,85}$ e/ou limite irregular ou turvo são características de suspeição de melanoma. ${ }^{67,70,79,81,86}$

A letra "C" se refere à mudança (change, em inglês) da lesão, seja um aumento recente ou súbito do tamanho, que se compara à fase radial de 
crescimento, ${ }^{70,84}$ ou, ainda, perda de homogeneidade do pigmento ${ }^{70,87}$ ou alteração na morfologia da unha, sugerindo malignidade. Pode haver distrofia da placa ou ulceração. Vale lembrar que distúrbios de pigmentação podem não estar presentes em melanomas amelanóticos. ${ }^{6,68,70,84,87-89}$

A letra " $D$ " representa o acometimento do dígito. A presença da faixa pigmentada em apenas um dedo aumenta a suspeição de $\mathrm{MU}$, quando comparado ao acometimento de múltiplos dedos. ${ }^{68,70,86,87}$ Esse dado é importante, uma vez que as lesões pigmentadas estão presentes em $100 \%$ dos americanos negros com mais de 50 anos.

A extensão do pigmento marrom ou preto para além da unha, nas pregas laterais ou proximal, é contemplada na letra "E" ${ }^{63,70,90}$ Dessa forma, se inclui aqui o sinal de Hutchinson. A extensão do pigmento para a borda livre da unha também é descrita como um sinal de suspeição de $M U^{63}$

A letra "F", por sua vez, é um lembrete para se investigar a história familiar ou pessoal de melanomas, já que isso aumenta o risco de desenvolvimento futuro de lesões malignas. ${ }^{70} \mathrm{Na}$ presença de dados positivos, a documentação fotográfica das lesões deve assessorar o seguimento. ${ }^{91}$ Espera-se que a abordagem sistemática das lesões favoreça o diagnóstico precoce. ${ }^{49}$

Segundo o trabalho de Di Chiacchio e colaboradores ${ }^{69}$, a aplicação da regra do $A B C D E F$ não influenciou a acurácia para o diagnóstico precoce do MU quando comparada ao exame clínico. Essa pesquisa estudou ainda a acurácia da dermatoscopia da placa ungueal e da dermatoscopia intraoperatória, sendo este último o único método que influenciou o diagnóstico correto.

A dermatoscopia da placa ungueal é um exame não invasivo auxiliar no diagnóstico de lesões ungueais melanocíticas. Além de reduzir o número de cirurgias desnecessárias e aumentar a precisão do diagnóstico clínico, pode auxiliar na escolha da técnica de biópsia mais adequada. ${ }^{92-98}$ Ainda que auxilie a determinar se o pigmento é melânico ou não, a dermatoscopia não é tão útil para diferenciação da proliferação melanocítica. ${ }^{9,99}$

A melanoníquia longitudinal de coloração marrom ou preta à 
dermatoscopia é suspeita de melanoma ou nevo melanocítico. Porém, conforme demonstrado histopatologicamente em recente estudo, a melanoníquia racial em pacientes com fototipo IV, $\mathrm{V}$ ou $\mathrm{VI}$, resultante de hipermelanose, também pode se apresentar à dermatoscopia com coloração marrom ou preta. ${ }^{100}$

Segundo consenso sobre dermatoscopia da placa ungueal em melanoníquias, a decisão de excisar a lesão deve se basear nos critérios clínicos (história e exame físico) e não nos padrões da dermatoscopia da placa. A dermatoscopia do hiponíquio é muito útil para o diagnóstico diferencial entre nevo e melanoma, nos casos de pigmentação do tecido periungueal. ${ }^{101} \mathrm{Um}$ padrão de linhas irregulares é a característica dermatoscópica mais proeminente do melanoma lentiginoso acral pigmentado do aparelho ungueal. A lesão amelanótica é caracterizada por restos de pigmentação e um padrão vascular polimórfico. ${ }^{102}$

A dermatoscopia da placa ungueal tem suas limitações por não analisar diretamente a matriz da unha, de onde se origina o pigmento. ${ }^{103}$ Nesse contexto, a dermatoscopia intraoperatória é uma nova técnica que supre a limitação da técnica anterior, oferecendo maior sensibilidade e especificidade no exame de lesões pigmentadas, pois é realizada diretamente na matriz, após avulsão da placa. ${ }^{99,104,105}$ Permite a visualização direta dos padrões dermatoscópicos da lesão pigmentada.

Hirata e colaboradores ${ }^{103}$ validaram quatro padrões de dermatoscopia intraoperatória da matriz ungueal para melanoníquia longitudinal: cinza regular (hipermelanose); marrom regular (hiperplasia melanocítica benigna); marrom regular com glóbulos ou manchas (nevo melanocítico); e irregular (melanoma). A técnica demonstrou $100 \%$ de sensibilidade e especificidade para o diagnóstico de nevo da matriz ungueal, minimizando cicatrizes desnecessárias no caso dessas lesões benignas.

Em contrapartida, não foram observadas as mesmas taxas de sensibilidade e especificidade no diagnóstico diferencial entre hiperplasia melanocítica e MU. O padrão irregular, caracterizado por linhas longitudinais de cores e espessuras irregulares, com ou sem glóbulos, foi observado em 13 dos 15 casos de melanoma, sendo 12 deles in situ. Esses dados conferem uma 
sensibilidade de $87 \%$ e especificidade de $100 \%{ }^{103}$

Mesmo com os critérios clínicos e as técnicas de dermatoscopia e dermatoscopia intraoperatória, a acurácia do dermatologista é relativamente deficiente e depende do diagnóstico histopatológico, especialmente nos casos controversos. $^{69,106}$

O exame histopatológico é o padrão ouro para a confirmação diagnóstica. Existem algumas técnicas para a realização da biópsia da matriz ungueal. Uma delas é a excisão longitudinal lateral na espessura total, permitindo a completa remoção da lesão melanocítica, na qual pode ocorrer distrofia da placa ungueal. Outra opção seria o punch de $3 \mathrm{~mm}$, que também pode causar distrofia, especialmente em crianças cujos dedos são pequenos, além da possibilidade de não remover a lesão por inteiro. Quaisquer dessas técnicas são consideradas adequadas para a obtenção de espécimes suficientes, garantindo a acurácia do diagnóstico histopatológico. ${ }^{72,99}$

Uma técnica que se destaca das anteriores por permitir a excisão completa da lesão sem distrofia permanente é a excisão tangencial. Descrita por Haneke e Baran ${ }^{59}$ essa técnica também fornece material suficiente para o exame histopatológico, mas requer técnicos e patologistas familiarizados. Não permite a medida precisa do índice de Breslow; porém, melanomas que se apresentam como melanoníquia longitudinal costumam ser in situ ou microinvasivos e o Breslow não é medido. ${ }^{99}$

A técnica de biópsia por excisão tangencial é realizada com bloqueio anestésico distal do dedo. Duas incisões oblíquas são realizadas na prega ungueal proximal, a fim de expor totalmente a placa ungueal. A seguir, a porção proximal da placa ungueal é rebatida expondo a matriz. A lesão é então delimitada delicadamente com a uma lâmina de bisturi na vertical; o espécime é removido tangencialmente e colocado sobre papel filtro, sendo encaminhado ao patologista, embebido em formol (Figura 2). 


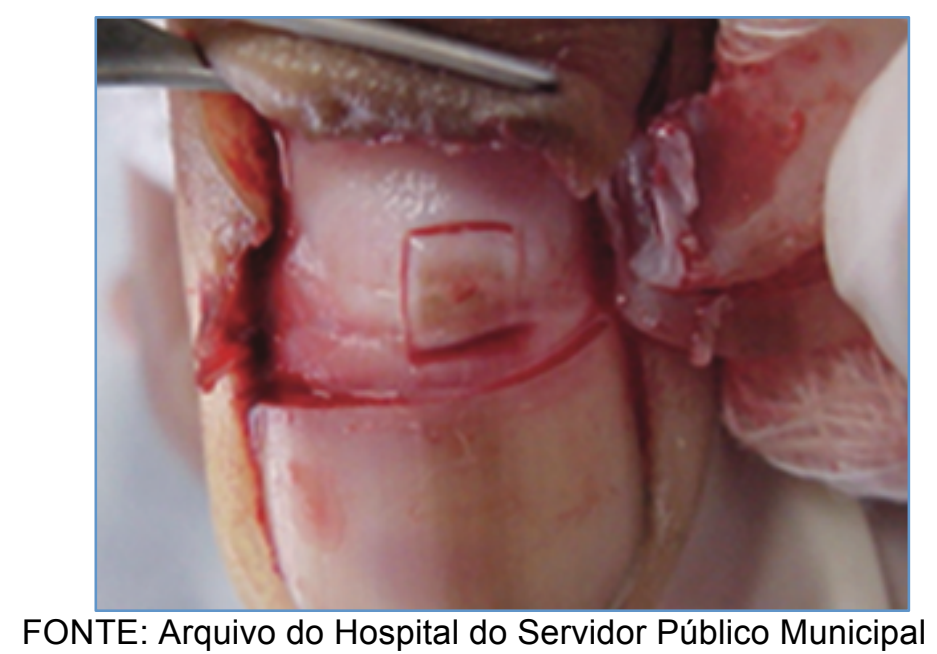

Figura 2 - Técnica de biópsia por excisão tangencial

\subsubsection{Histopatologia}

A classificação do melanoma em suas diferentes formas baseia-se na identificação da fase de crescimento radial (extensivo superficial, lentigo maligno melanoma, melanoma acral) ou na sua ausência (melanoma nodular). ${ }^{107}$

Os subtipos histológicos mais comuns de melanoma subungueal são 0 lentiginoso acral e o nodular. ${ }^{6,50,51} \mathrm{~A}$ maioria dos melanomas se inicia na epiderme, como melanoma in situ, depois se expandem lateralmente (fase de crescimento radial), tornam-se localmente invasivos (fase de crescimento vertical) e também metastáticos. ${ }^{107}$

As lesões in situ podem simular um padrão benigno, especialmente na periferia, com aumento nos melanócitos basais e na hiperpigmentação com atipia apenas focal dos melanócitos. Entretanto, no centro da lesão, encontrase atipia citológica intensa e uniforme. A pigmentação é pronunciada, resultando da presença de melanófagos na derme superior e de grandes agregados de melanina na camada córnea. ${ }^{108}$

A presença de intensa inflamação caracterizada por linfócitos e macrófagos na junção dermo-epidérmica, em meio a células de melanoma intraepidérmicas, pode ser a única pista histológica do melanoma lentiginoso acral em fase de crescimento radial. ${ }^{64} \mathrm{~A}$ região basal pode revelar aumento no 
número de grandes melanócitos, com núcleos aumentados e nucléolos proeminentes.

A característica mais importante é a presença de melanócitos geralmente pigmentados, com longos dendritos e que atravessam uma distância equivalente a três a quatro queratinócitos adjacentes. Os melanócitos anormais podem formar grandes ninhos que se projetam na derme e se estendem profusamente ao longo de ductos écrinos. ${ }^{63}$

A maioria dos melanomas ungueais inicia-se na matriz; entretanto, pode surgir no leito unguel devido a melanócitos quiescentes desse local. Quando o melanoma origina-se da matriz, a melanoniquia longitudinal está presente em cerca de $76 \%$ dos casos. Além disso, o melanoma da matriz pode invadir outras partes da unidade ungueal, ocasionando pigmentação da dobra ungual com ou sem o sinal de Hutchinson e distrofia da unha. Quando surge do leito ungueal, o melanoma tipicamente apresenta-se como um nódulo subungueal não pigmentado usualmente acompanhado de onicólise. ${ }^{109}$

\subsubsection{Tratamento}

O tratamento do melanoma ungueal é cirúrgico, ainda que não haja consenso absoluto acerca da melhor técnica para a sua abordagem. Mas é unânime que a excisão completa da lesão seja realizada o mais precoce possível.

Historicamente, esse tumor tem sido removido em bloco, como produto da amputação do dígito acometido. Essa técnica já foi muito difundida como a melhor na prevenção da recorrência e redução do óbito por metástases. No entanto, a comparação de diferentes níveis de amputação não demonstrou vantagem da amputação metacarpal sobre a matacarpofalangeana, interfalangeana proximal ou distal. ${ }^{18,20,22,110-113}$

Desse modo, notou-se que nos últimos 30 anos a evolução do paciente não é determinada pela radicalidade da amputação, mas pelo momento em que a cirurgia é realizada. ${ }^{6,74,77,114,115-118}$ Quando diagnosticado no início, o MU 
ainda é in situ ou minimamente invasivo, isto é, a invasão corresponde à fase de crescimento radial com Breslow < 0,5 mm. ${ }^{1}$ Abordagens mais conservadoras, como a excisão local, foram propostas para esses casos, com resultado curativo. ${ }^{14,24,119}$ Isso tornou o tratamento adequado do $\mathrm{MU}$, além de desafiador, um tema controverso.

\subsubsection{Amputação}

A amputação começou a ser difundida por Hutchinson em 1886 como terapia de escolha para o MU. Já em sua descrição original, ele a introduzia de forma precoce e mandatória. ${ }^{46} \mathrm{~A}$ cirurgia radical era recomendada nos ossos metacarpo/metatarso ${ }^{120}$ ou nas articulações metacarpo-falangeana ${ }^{121}$ ou metatarso-falangeana. ${ }^{120,122,123}$

Amputações dos dedos, particularmente do polegar, implicam prejuízo funcional e estético. Amputações moderadas foram propostas a fim de preservar os dedos, sem comprometer as margens de segurança. Algumas vezes, defendia-se a manutenção da cabeça do primeiro metatarso, para manter a marcha fisiológica. ${ }^{122,123}$ Por outro lado, após recorrências locorregionais, alguns autores chegaram a propor amputações mais radicais, como a desarticulação do quadril. ${ }^{120,124}$

O primeiro estudo a revisar o tratamento do MU foi em 1965. Trinta e quatro pacientes foram submetidos à amputação da articulação interfalangeana ou proximalmente. Os três pacientes tratados com a amputação menos agressiva (na articulação interfalangeana) apresentaram recorrência e não sobreviveram a 5 anos. Diante desse achado, a amputação local foi considerada inadequada para tratar o MU. Porém, a profundidade das lesões não foi considerada em tal conclusão. Além disso, 17 dos 34 pacientes desenvolveram recidiva ou metástase linfonodal. Então, se metade dos pacientes apresentaram evolução da doença, possivelmente isso seria resultado do próprio melanoma, e a conclusão a respeito da técnica cirúrgica seria infundada. ${ }^{125}$ 
Em 1992, Park e colaboradores ${ }^{117}$ demonstraram que não houve comprometimento da sobrevida de pacientes tratados com amputação distal ou excisão local, quando comparados aos submetidos à amputação proximal. Observaram que nos pacientes com excisões mais localizadas houve tendência à recorrência precoce, mas não local e sim linfonodal, o que não parece estar associado ao tipo de cirurgia escolhida. Nesse estudo, a técnica da excisão local não foi esclarecida. ${ }^{117}$

Posteriormente, Heaton ${ }^{126}$ também confirmou que o nível de amputação não afeta a recidiva local, tampouco a sobrevida dos pacientes, em um estudo com 46 pacientes tratados com diferentes abordagens cirúrgicas. Da mesma forma, Finley ${ }^{74}$ pesquisou 22 pacientes com MU, dos quais, 7 foram tratados com amputação distal sem recidiva, reafirmando a conclusão dos trabalhos anteriores. A evolução do nível de amputação pode ser observada na Figura 3.

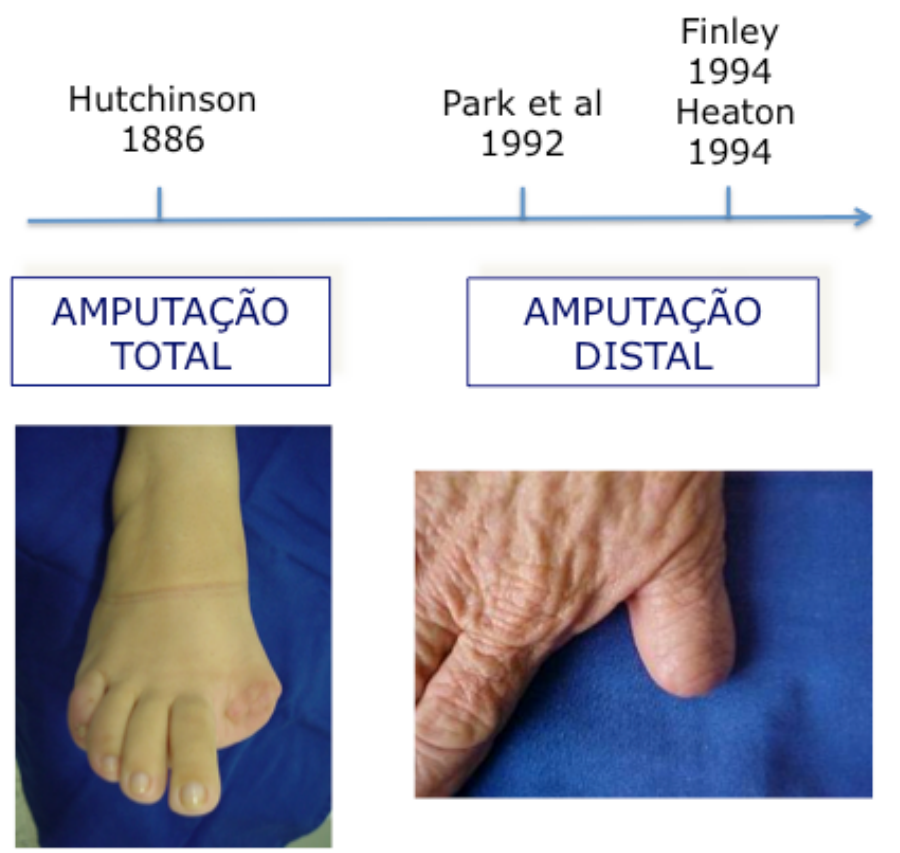

Figura 3 - Evolução do nível de amputação

Estudos mostram que o nível de amputação não afeta a sobrevida nem o risco de recidiva local. ${ }^{117,127-129}$ Os dados sugerem, portanto, que o nível de amputação não é um parâmetro prognóstico para a sobrevida livre de doença e incentivam abordagens conservadoras. ${ }^{16,50,52,74,130}$ 


\subsubsection{Cirurgia funcional}

Em 2002, Clarkson e colaboradores ${ }^{26}$ descreveram um caso com dois melanomas ungueais in situ, na mesma unha. O leito ungueal foi excisado até o osso, seguido de reconstrução com retalho do dedo médio. Ainda que não tenha detalhado a técnica, esse artigo elucidou a abordagem conservadora de lesões precocemente diagnosticadas. ${ }^{26}$

Foi em 2003 que Moehrle e colaboradores ${ }^{27}$ nomearam a remoção do complexo ungueal com preservação da falange distal de "cirurgia funcional" e a descreveram tecnicamente. Essa cirurgia corresponde à remoção de todo o complexo ungueal com retirada do tumor com margem de segurança e ressecção parcial da porção distal da falange distal (processus unguinatus). 0 tumor clinicamente visível é removido em bloco, juntamente com o leito e a matriz ungueal, com margem lateral de $5 \mathrm{~mm}$. Nessa descrição, o defeito cirúrgico foi coberto com enxerto cutâneo, procedimento realizado sob anestesia local e torniquete. ${ }^{27}$

No estudo de Moehrle e colaboradores ${ }^{27}, 31$ pacientes foram submetidos à amputação e comparados com 31 pacientes tratados com a cirurgia funcional. A sobrevida em 5 anos encontrada no primeiro grupo foi de $66,5 \%$ contra $91,7 \%$ no segundo. Não houve diferença na taxa de recidiva entre os dois grupos. Porém, nessas amostras, foram incluídos pacientes com estádio I e II, de modo que não há casos in situ (estádio 0 ), segundo a classificação da American Joint Committee on Cancer (AJCC). ${ }^{131}$

Em 2005, Lazar e colaboradores ${ }^{132}$, baseados na premissa de que a recidiva local e a sobrevida não são afetadas pelo nível de amputação, trataram 9 pacientes com $\mathrm{MU}$ in situ, por meio da remoção completa do aparelho ungueal sem excisar o periósteo. Não foi observada recidiva dentre os pacientes, com tempo de seguimento variando entre 4 meses a 7 anos.

Rayatt e colaboradores ${ }^{130}$ trataram quatro pacientes com MU, cujo Breslow variava de $0,9 \mathrm{~mm}$ a $4 \mathrm{~mm}$, com a excisão do tumor, incluindo o periósteo, com margem de pelo menos $1 \mathrm{~cm}$, com o consentimento dos pacientes. Houve recidiva do caso em que o tumor era mais espesso $(4 \mathrm{~mm})$. 
Concluiu-se que essa abordagem é desaconselhada em tumores espessos, ficando reservada de forma encorajadora para tumores finos, com manutenção funcional.

Outros autores obtiveram sucesso terapêutico com a técnica, não apresentando recidiva, e concluíram que essa abordagem cirúrgica está indicada no tratamento do $\mathrm{MU}$ in situ, com bom resultado cosmético e funcional, sem alterar o prognóstico. ${ }^{1,26-30}$ Até 2011, verificou-se que, dos 29 casos de melanoma in situ tratados com a cirurgia conservadora na literatura, 3 foram submetidos à amputação por margem positiva e outros 2 recidivaram. ${ }^{1}$

Nakamura e colaboradores ${ }^{133}$, com a finalidade de investigar a possibilidade de tratar casos invasivos com cirurgia conservadora, mediram a distância do tumor até a superfície óssea de 30 espécimes cirúrgicos de MU invasivo. Desses, quatro apresentavam contato com o osso. Em todos os casos com espessura $<4 \mathrm{~mm}$, a distância do tumor até o osso era de no mínimo $0,9 \mathrm{~mm}$. Dos nove casos não ulcerados, um invadia o osso e nos casos com espessura $>4 \mathrm{~mm}$ o contato ou a invasão óssea eram maiores. ${ }^{133}$

Portanto, demonstrou-se que nem todos os melanomas ungueais invasivos estão adjuntos ou invadem a superfície óssea da falange distal nos espécimes amputados. Concluíram que a cirurgia conservadora é uma possibilidade para tumores com espessura intermediária (de 1,01 $\mathrm{mm}$ até $4 \mathrm{~mm}$ ), porém também consideraram que há pouca evidência científica demonstrando a sobrevida dos casos invasivos tratados de maneira conservadora. ${ }^{133}$

Uma variante da cirurgia funcional foi descrita em 2013 para tratar um caso in situ ${ }^{134}$ e em 2014 em um caso com 0,95 mm de espessura. ${ }^{135}$ Além de remover todo o aparelho ungueal em bloco, essa técnica utiliza um instrumento cirúrgico específico para remover uma camada óssea da falange distal, paralelamente à unha. Essa variante preserva a funcionalidade e permite o controle da margem profunda, ao produzir uma amostra que pode ser avaliada pelo patologista. ${ }^{134,135}$ Como desvantagem, destaca-se o risco de comprometer a articulação interfalangeana distal durante a dissecção da êntese. ${ }^{135}$

Chow e colaboradores ${ }^{134}$ advogam o uso dessa variante técnica para casos de MU in situ. Maekawa ${ }^{135}$ defende a aplicabilidade da técnica para casos em que o grau de invasão não está bem estabelecido no pré-operatório. 
Ambos os trabalhos concordam que os tumores invasivos requerem intervenção mais agressiva.

Como não há diretrizes ou protocolos que guiem o manejo cirúrgico desse tumor, as evidências baseadas na experiência de poucos são de grande valia para a evolução da técnica (Figura 4).
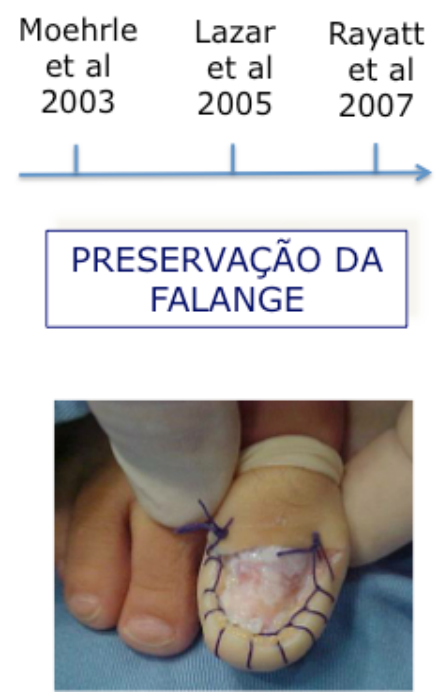

FONTE: Arquivo do Hospital do Servidor Público Municipal Figura 4 - Técnica conservadora, com preservação da falange

\subsubsection{Prognóstico}

Aproximadamente $20 \%$ dos pacientes com MU se encontram na fase I da classificação de estadiamento TNM no momento do diagnóstico, contra $80 \%$ dos pacientes com melanoma cutâneo ao diagnóstico, conforme Figura 5. .,74,87 $^{-3}$ 


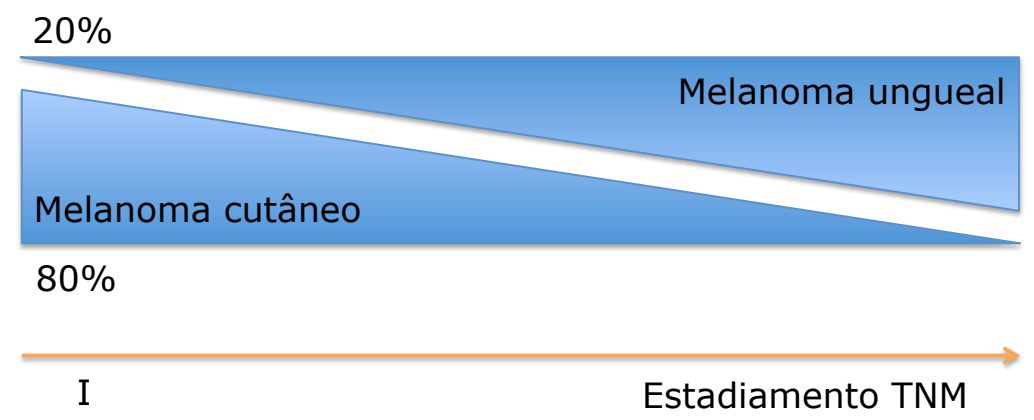

Figura 5 - Representação gráfica da distribuição dos pacientes com melanoma ungueal e cutâneo na fase I de estadiamento TNM.

Devido à dificuldade no diagnóstico precoce, o MU possui prognóstico reservado e baixa sobrevida quando comparado às demais formas de melanoma. A interferência da placa ungueal alterando a reflexão da luz e o aspecto amelanótico da lesão (presente em 15\%-65\% dos casos) são fatores que contribuem para a detecção tardia. ${ }^{12-14}$

Características clinicopatológicas, como idade avançada no momento do diagnóstico, elevado número de mitoses dérmicas, presença de ulceração, lesão amelanótica, e grau avançado da doença, foram relacionadas a um prognóstico desfavorável. ${ }^{6,52,77}$

A sobrevida dos pacientes em 5, 10 e 20 anos é relatada em $60,5 \%$, $43,8 \%$, e $28,3 \%$, respectivamente..$^{50} \mathrm{Em}$ alguns artigos, a sobrevida em 5 anos chega a variar entre $18 \%$ a $58 \% .{ }^{50,52,136}$

Para abordar tamanha agressividade, o tratamento deve ser cirúrgico e garantir a extirpação do tumor. 


\section{OBJETIVOS}

\subsection{Objetivo Primário}

O presente estudo tem como objetivo avaliar comparativamente a sobrevida dos pacientes com MU tratados com cirurgia funcional ou amputação distal.

\subsection{Objetivo Secundário}

Como objetivo secundário, pretende-se avaliar se características histopatológicas, como nível de invasão, presença de mitose e ulceração ao histopatológico influenciam a sobrevida. 


\section{MATERIAL E MÉTODOS}

\subsection{Desenho do Estudo}

O trabalho foi um estudo de coorte retrospectiva.

\subsection{Casuística}

Foram incluídos no estudo pacientes portadores de MU tratados cirurgicamente no Hospital do Servidor Público Municipal ou em clínica particular, no período de 2003 a 2013. Os casos foram tratados por um de dois cirurgiões, um dermatológico e um oncológico. Considerou-se como critério de inclusão o mínimo de 2 anos de seguimento. Analisaram-se: sexo e idade dos pacientes; características do tumor (localização anatômica, nível de invasão, presença de mitose, ulceração ou regressão ao histopatológico e comprometimento de linfonodo sentinela); técnica cirúrgica adotada; tempo de seguimento após a cirurgia; e desfecho (sem evidência de doença, recidiva local, metástase e óbito) (Quadro 2).

Inicialmente, os casos foram divididos em dois grupos, isto é, um de pacientes tratados com amputação distal e outro composto por pacientes tratados com cirurgia funcional, facilitando a caracterização de cada um deles, conforme Tabela 1. Porém, em virtude da pequena amostra, foi necessário agrupar todos os casos, para viabilizar a análise estatística. 
Quadro 2 - Casos de melanoma ungueal estudados entre 2003 e 2013

\begin{tabular}{|c|c|c|c|c|c|}
\hline Paciente & Sexo & $\begin{array}{l}\begin{array}{l}\text { Idade } \\
\text { (anos) }\end{array} \\
\end{array}$ & Localização & $\begin{array}{l}\text { Invasão } \\
\text { tumoral }\end{array}$ & $\begin{array}{c}\text { Cirurgia } \\
\text { realizada }\end{array}$ \\
\hline 1 & $\mathrm{~F}$ & 56 & Hálux & Tis & Funcional \\
\hline 2 & M & 29 & Hálux & Tis & Funcional \\
\hline 3 & $\mathrm{~F}$ & 25 & $4^{\circ} \mathrm{QD}$ & Tis & Funcional \\
\hline 4 & M & 5 & Hálux & Tis & Funcional \\
\hline 5 & $\mathrm{~F}$ & 69 & $1^{\circ} \mathrm{QD}$ & Tis & Amputação \\
\hline 6 & M & 42 & $3^{\circ} \mathrm{QD}$ & Tis & Funcional \\
\hline 7 & M & 59 & Hálux & $2,5 \mathrm{~mm}$ & Funcional \\
\hline 8 & $\mathrm{~F}$ & 49 & $1^{\circ} \mathrm{QD}$ & $3,5 \mathrm{~mm}$ & Funcional \\
\hline 9 & $\mathrm{~F}$ & 32 & $4^{\circ} \mathrm{QD}$ & Tis & Funcional \\
\hline 10 & $\mathrm{~F}$ & 81 & Hálux & Tis & Funcional \\
\hline 11 & $F$ & 47 & Hálux & 12 mm & Amputação \\
\hline 12 & $F$ & 68 & $4^{\circ} \mathrm{PD}$ & $5 \mathrm{~mm}$ & Amputação \\
\hline 13 & M & 49 & Hálux & Tis & Amputação \\
\hline 14 & M & 77 & Hálux & $4,35 \mathrm{~mm}$ & Amputação \\
\hline 15 & M & 39 & Hálux & $0,31 \mathrm{~mm}$ & Funcional \\
\hline 16 & M & 49 & Hálux & Tis & Amputação \\
\hline 17 & M & 62 & Hálux & Tis & Amputação \\
\hline 18 & $\mathrm{~F}$ & 56 & $1^{\circ} \mathrm{QD}$ & Tis & Funcional \\
\hline 19 & $\mathrm{~F}$ & 65 & Hálux & $2,65 \mathrm{~mm}$ & Amputação \\
\hline 20 & $\mathrm{~F}$ & 69 & $4^{\circ} \mathrm{QD}$ & Tis & Funcional \\
\hline 21 & $\mathrm{~F}$ & 67 & $5^{\circ} \mathrm{QD}$ & $1,9 \mathrm{~mm}$ & Amputação \\
\hline
\end{tabular}

NOTA: F: feminino; M: masculino; P: pododáctilo; Q: quirodáctilo;

D: direito; Tis: tumor in situ. 
Tabela 1 - Características clínicas e histopatológicas dos casos, estratificados por cirurgia funcional e amputação

\begin{tabular}{|c|c|c|c|}
\hline Características & Cirurgia funcional & Amputação & TOTAL \\
\hline Pacientes & 12 & 9 & 21 \\
\hline \multicolumn{4}{|l|}{ Sexo } \\
\hline Masculino & 5 & 4 & 9 \\
\hline Feminino & 7 & 5 & 12 \\
\hline \multicolumn{4}{|l|}{ Idade } \\
\hline$<60$ anos & 10 & 3 & 13 \\
\hline$>60$ anos & 2 & 6 & 8 \\
\hline \multicolumn{4}{|l|}{ Breslow (espessura) } \\
\hline \multicolumn{4}{|l|}{ Média } \\
\hline In situ (Tis) & 9 & 4 & 13 \\
\hline$<=1 \mathrm{~mm}(\mathrm{~T} 1)$ & 1 & 0 & 1 \\
\hline$>1 \mathrm{~mm}$ a $<=2 \mathrm{~mm}(\mathrm{~T} 2)$ & 0 & 1 & 1 \\
\hline$>2 \mathrm{~mm}$ a $<=4 \mathrm{~mm}$ (T3) & 2 & 1 & 3 \\
\hline$>4 \mathrm{~mm}(\mathrm{~T} 4)$ & 0 & 3 & 3 \\
\hline \multicolumn{4}{|l|}{ Ulceração histopatológica } \\
\hline Presente & 0 & 4 & 4 \\
\hline Ausente & 12 & 5 & 17 \\
\hline \multicolumn{4}{|l|}{ Mitose } \\
\hline Presente & 2 & 4 & 6 \\
\hline Ausente & 10 & 5 & 15 \\
\hline \multicolumn{4}{|l|}{ Linfonodo sentinela } \\
\hline Positivo & 0 & 1 & 1 \\
\hline Negativo & 1 & 5 & 6 \\
\hline Não realizado & 11 & 3 & 14 \\
\hline \multicolumn{4}{|l|}{ Regressão } \\
\hline Presente & 0 & 1 & 1 \\
\hline Ausente & 12 & 8 & 20 \\
\hline \multicolumn{4}{|l|}{ Extremidade } \\
\hline Mão & 6 & 2 & 8 \\
\hline Pé & 6 & 7 & 13 \\
\hline \multicolumn{4}{|l|}{ Desfecho } \\
\hline Sem evidência de doença & 9 & 7 & 16 \\
\hline Óbito por metástase & 0 & 1 & 1 \\
\hline $\begin{array}{l}\text { Metástase linfonodal em } \\
\text { tratamento }\end{array}$ & 1 & 1 & 2 \\
\hline Recidiva local & 2 & 0 & 2 \\
\hline
\end{tabular}




\subsection{Procedimento}

Os parâmetros clínicos analisados no estudo foram coletados a partir da revisão de prontuários. Da mesma forma, foram avaliados os exames laboratoriais, como hemograma e LDH, e exames de imagem, como ultrassom de abdome e raios- $X$ de tórax periódicos realizados por todos os pacientes.

Foram incluídos casos de MU confirmados por biópsia e operados por um de dois cirurgiões, um dermatológico e outro oncológico.

A escolha da técnica foi determinada pelo nível de invasão tumoral e pelo consentimento do paciente, que, em algumas situações, optou pela funcional em detrimento da perda da funcionalidade, ainda que tivesse um tumor mais espesso. As lâminas histológicas foram revisadas por um dermatopatologista para confirmar o diagnóstico de melanoma.

Os pacientes foram classificados de acordo com as intercorrências que apresentaram durante sua evolução, seja recidiva local, metástase a distância ou óbito por metástase. Essas variáveis foram denominadas "desfecho". Para fazer a análise de sobrevida, todos os desfechos foram agrupados em uma categoria (evento). Sendo assim, foi analisado o tempo até a ocorrência de algum evento (metástase, recidiva ou óbito).

\subsection{Análise Estatística}

Foi realizada análise exploratória de dados por meio de medidas resumo (média, desvio padrão, mínimo, mediana e máximo). O modelo de riscos proporcionais de Cox foi usado para avaliar a sobrevida dos pacientes. $\mathrm{Na}$ análise múltipla, o critério de seleção de variáveis stepwise foi usado para encontrar os fatores de risco que atuam em conjunto na sobrevida.

O nível de significância considerado nas análises foi de $5 \%$.

O software utilizado foi SAS 9.4 e R 3.0.2. 


\section{RESULTADOS}

\subsection{Análise Descritiva - Caracterização da Amostra}

Foram estudados 21 pacientes, e o tempo médio de acompanhamento foi de 73 meses, variando de 24 a 175 meses, conforme Tabela 2.

Tabela 2 - Medidas de posição e dispersão da idade e do tempo de seguimento dos pacientes

\begin{tabular}{lcccccc}
\hline Variável & $\mathbf{N}$ & Média & DP & Mínimo & Mediana & Máximo \\
\hline Idade & 21 & 52,14 & 18.79 & 5.00 & 56.00 & 81.00 \\
$\begin{array}{l}\text { Tempo de } \\
\begin{array}{l}\text { Seguimento } \\
\text { (meses) }\end{array}\end{array}$ & 21 & 73,95 & 46.94 & 24.00 & 52.00 & 175.00 \\
\hline
\end{tabular}

Há predomínio do sexo feminino $(57,14 \%)$ sobre o masculino $(42,86 \%)$. Em relação à localização, 12 casos $(57,14 \%)$ acometem o hálux, e nove $(42,86 \%)$ estão distribuídos nos outros dedos, da seguinte forma: três no primeiro quirodáctilo; um no terceiro quirodáctilo; três no quarto quirodáctilo; um no quarto pododáctilo; e um no quinto pododáctilo.

Em relação ao nível de invasão tumoral, 13 casos eram in situ; um possuía $0,31 \mathrm{~mm}$; e sete variaram entre $1,9 \mathrm{~mm}$ a $12 \mathrm{~mm}$, este último invadia o periósteo.

A amputação foi executada em nove casos, na falange distal, enquanto os outros 12 foram tratados com cirurgia funcional $(57,14 \%)$. Em relação ao desfecho, 16 pacientes não apresentaram evidência de doença durante 0 seguimento, que variou de 24 a 175 meses.

Dois dos 12 pacientes submetidos à funcional tiveram recidiva local, após 11 e 77 meses. O primeiro apresentava um melanoma in situ e foi reabordado com a mesma técnica, estando sem recidiva. O segundo foi submetido à amputação devido ao tumor invasivo e encontra-se sem sinais de atividade nos últimos 24 meses. 
Outro paciente tratado com funcional apresentou metástase em linfonodo inguinal após 18 meses da cirurgia e encontra-se em tratamento.

Dois dos nove pacientes amputados também apresentaram metástase. Um em linfonodo mediastinal e tronco celíaco após 24 meses, sendo tratado com quimioterapia e imunoterapia, no momento sem sinais de recidiva. $O$ outro teve metástase pulmonar após 26 meses da cirurgia e óbito após 2 meses. Os demais pacientes (16) não apresentaram nenhuma evidência de doença durante a evolução e constituem $76,19 \%$ da amostra. As características dos pacientes, assim como os defechos podem ser podem ser observados no Quadro 3.

De acordo com as características histopatológicas, foi observada regressão em apenas um caso, ulceração em quatro casos e mitose em seis casos. A biópsia sentinela foi positiva em um caso, negativa em seis e não realizada em 14 casos. O Quadro 4 apresenta as variáveis distribuídas em frequência e porcentagem.

Quadro 3 - Características dos pacientes e respectivos desfechos

\begin{tabular}{|c|c|c|c|c|c|c|c|}
\hline Paciente & Sexo & $\begin{array}{l}\text { Idade } \\
\text { (anos) }\end{array}$ & Localização & $\begin{array}{l}\text { Invasão } \\
\text { tumoral }\end{array}$ & $\begin{array}{l}\text { Cirurgia } \\
\text { realizada }\end{array}$ & $\begin{array}{c}\text { Tempo de } \\
\text { seguimento } \\
\text { pós-cirurgia } \\
\text { (meses) }\end{array}$ & $\begin{array}{l}\text { Desfecho } \\
\text { (SED/recidival } \\
\text { metástase/ } \\
\text { óbito) }\end{array}$ \\
\hline 1 & $F$ & 56 & Hálux & Tis & Funcional & 28 & SED \\
\hline 2 & $M$ & 29 & Hálux & Tis & Funcional & 39 & SED \\
\hline 3 & $\mathrm{~F}$ & 25 & $4^{\circ} \mathrm{QD}$ & Tis & Funcional & 114 & SED \\
\hline 4 & M & 5 & Hálux & Tis & Funcional & 142 & SED \\
\hline 5 & $\mathrm{~F}$ & 69 & $1^{\circ} \mathrm{QD}$ & Tis & Amputação & 175 & SED \\
\hline 6 & M & 42 & $3^{\circ} \mathrm{QD}$ & Tis & Funcional & 101 & Recidiva \\
\hline 7 & $M$ & 59 & Hálux & $2,5 \mathrm{~mm}$ & Funcional & 32 & Metástase \\
\hline 8 & $\mathrm{~F}$ & 49 & $1^{\circ} \mathrm{QD}$ & $3,5 \mathrm{~mm}$ & Funcional & 127 & SED \\
\hline 9 & $\mathrm{~F}$ & 32 & $4^{\circ} \mathrm{QD}$ & Tis & Funcional & 42 & SED \\
\hline 10 & $\mathrm{~F}$ & 81 & Hálux & Tis & Funcional & 38 & Recidiva \\
\hline 11 & $F$ & 47 & Hálux & $12 \mathrm{~mm}$ & Amputação & 20 & SED \\
\hline 12 & $\mathrm{~F}$ & 68 & $4^{\circ} \mathrm{PD}$ & $5 \mathrm{~mm}$ & Amputação & 52 & SED \\
\hline 13 & M & 49 & Hálux & Tis & Amputação & 118 & SED \\
\hline 14 & $\mathrm{M}$ & 77 & Hálux & $4,35 \mathrm{~mm}$ & Amputação & 102 & SED \\
\hline 15 & $M$ & 39 & Hálux & $0,31 \mathrm{~mm}$ & Funcional & 38 & SED \\
\hline 16 & M & 49 & Hálux & Tis & Amputação & 116 & SED \\
\hline 17 & M & 62 & Hálux & Tis & Amputação & 26 & SED \\
\hline 18 & $\mathrm{~F}$ & 56 & $1^{\circ} \mathrm{QD}$ & Tis & Funcional & 28 & SED \\
\hline 19 & $\mathrm{~F}$ & 65 & Hálux & $2,65 \mathrm{~mm}$ & Amputação & 28 & Óbito \\
\hline 20 & $\mathrm{~F}$ & 69 & $4^{\circ} \mathrm{QD}$ & Tis & Funcional & 103 & SED \\
\hline 21 & $\mathrm{~F}$ & 67 & $5^{\circ} \mathrm{QD}$ & $1,9 \mathrm{~mm}$ & Amputação & 63 & Metástase \\
\hline
\end{tabular}

NOTA: F: feminino; M: masculino; P: pododáctilo; Q: quirodáctilo; D: direito; Tis: tumor in situ; SED: sem evidência de doença. 
Quadro 4 - Distribuição em frequência e porcentagem das variáveis categóricas

\begin{tabular}{|lcrc|}
\hline Variável & Categoria & $\begin{array}{c}\text { Estudo } \\
\text { (N) }\end{array}$ & $\begin{array}{c}\text { Porcentagem } \\
(\%)\end{array}$ \\
\hline \multirow{2}{*}{ Sexo } & Feminino & 12 & 57,14 \\
& Masculino & 9 & 42,86 \\
Localização & Hálux & 12 & 57,14 \\
& Não hálux & 9 & 42,86 \\
Invasão tumoral & Tis & 13 & 61,90 \\
& $>0$ mm & 8 & 38,10 \\
Cirurgia & Amputação & 9 & 42,86 \\
& Funcional & 12 & 57,14 \\
& Metástase & 2 & 9,52 \\
Desfecho & Recidiva & 2 & 9,52 \\
& Óbito por metástase & 1 & 4,76 \\
& Sem evidência de & 16 & 76,19 \\
Evento & doença & 16 & 76,19 \\
& 0 & 5 & 23,81 \\
Regressão & 1 & 20 & 95,24 \\
\multirow{2}{*}{ Ulceração } & Ausente & 1 & 4,76 \\
& Presente & 17 & 80,95 \\
Mitose & Ausente & 4 & 19,05 \\
& Presente & 15 & 71,43 \\
Biópsia sentinela & Ausente & 6 & 28,57 \\
& Presente & 6 & 28,57 \\
& Negativa & 1 & 6,76 \\
\hline
\end{tabular}

NOTA: Tis: tumor in situ.

A Tabela 3 mostra os resultados da regressão simples de Cox, ou seja, quando a influência no tempo para evento foi estudada individualmente para cada fator.

Tabela 3 - Resultados da regressão simples de Cox

\begin{tabular}{llcccc}
\hline \multicolumn{1}{c}{ Variável } & Cat risco & p-valor & $\begin{array}{c}\text { Razão de } \\
\text { riscos }\end{array}$ & IC de $\mathbf{9 5 \%}$ da & RR \\
\hline Idade & Contínua & 0,1861 & 1,039 & 0,982 & 1,101 \\
Sexo & F & 0,6921 & 1,437 & 0,239 & 8,661 \\
Localização & Não hálux & 0,5557 & 0,583 & 0,097 & 3,512 \\
Invasão & $>0$ mm & 0,2002 & 3,255 & 0,535 & 19,796 \\
Cirurgia & Amputação & 0,9362 & 0,929 & 0,154 & 5,610 \\
Ulceração & Presente & 0,1331 & 3,978 & 0,656 & 24,113 \\
Mitose & Presente & 0,0511 & 6,120 & 0,992 & 37,769 \\
\hline
\end{tabular}

A partir desse quadro, nota-se que nenhuma das variáveis estudadas apresentou influência significativa no tempo para a ocorrência de algum evento, uma vez que todos os p-valores são maiores que 0,05 . Porém, "mitose" está 
próximo ao nível de significância, indicando que pacientes com mitose apresentam maior risco de algum evento.

$\mathrm{Na}$ análise múltipla (multivariada), nenhuma variável se mostrou importante para explicar o tempo de ocorrência do evento, ou seja, nenhuma foi significativa também.

Os gráficos a seguir, de Kaplan-Meier, apresentam as curvas de sobrevivência global (Figura 6) e também pelas categorias das variáveis estudadas (Figuras 7 a 13). Na regressão de Cox, a idade foi avaliada como variável contínua; porém, para possibilitar a construção do gráfico, essa variável foi categorizada pela mediana (56 anos).

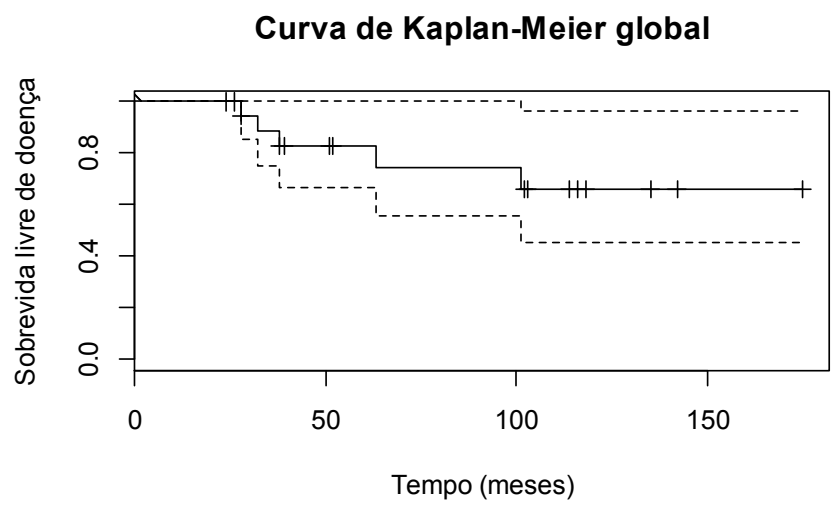

Figura 6 - Curva de sobrevivência global

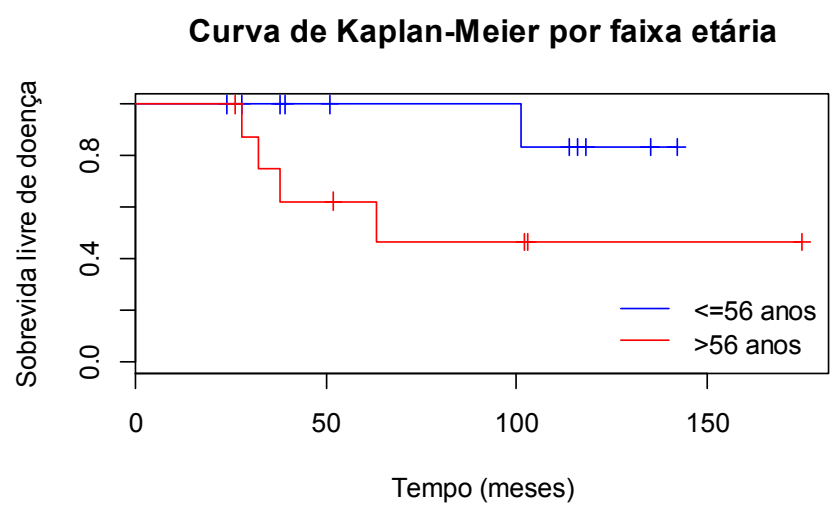

Figura 7 - Correlação entre sobrevida livre de doença e a faixa etária ( $<=56$ anos ou $>56$ anos) 


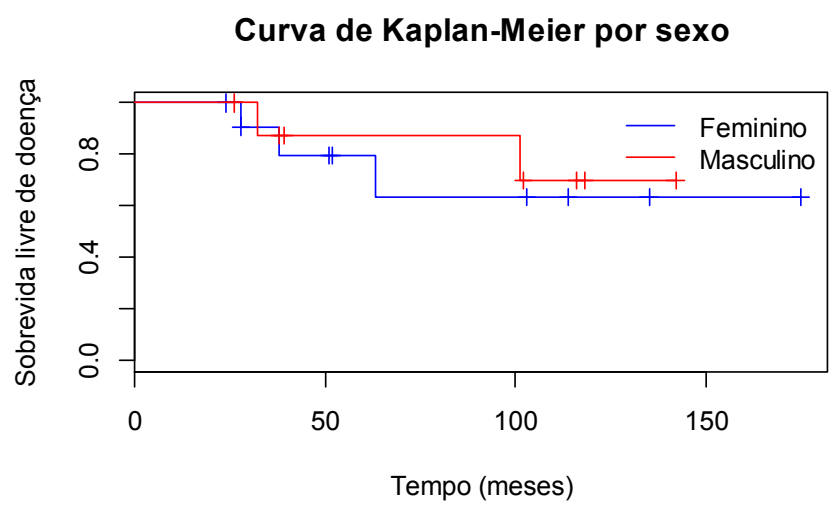

Figura 8 - Correlação entre sobrevida livre de doença e o gênero

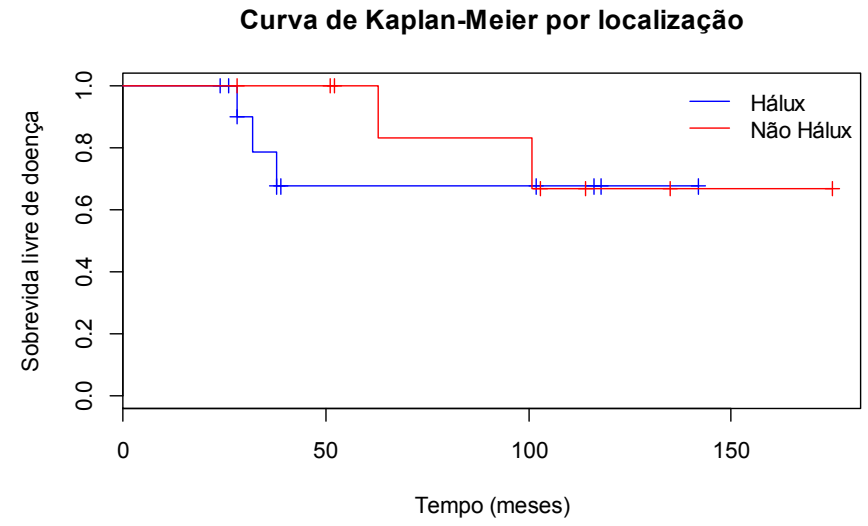

Figura 9 - Correlação entre sobrevida livre de doença e a localização, seja no hálux ou nos demais dedos agrupados como "não hálux"

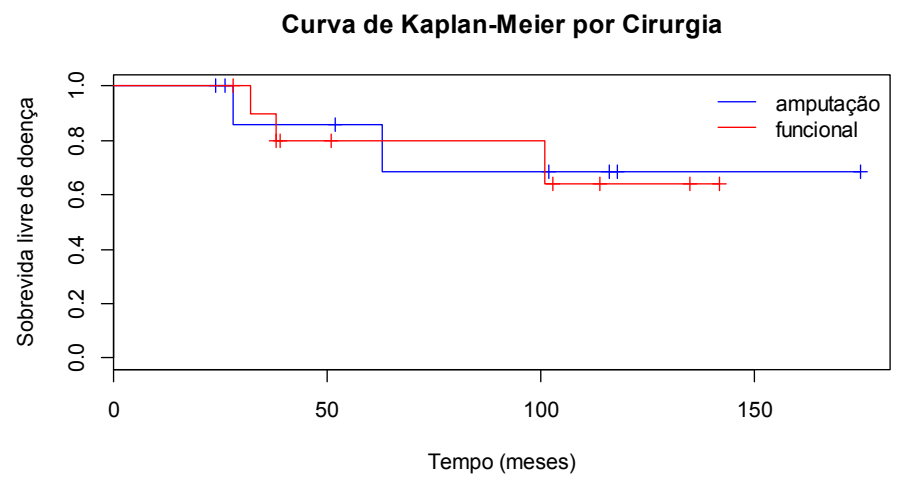

Figura 10 - Correlação entre sobrevida livre de doença e o seguimento dos pacientes de acordo com a técnica cirúrgica adotada para tratamento do melanoma ungueal (amputação versus cirurgia funcional) 


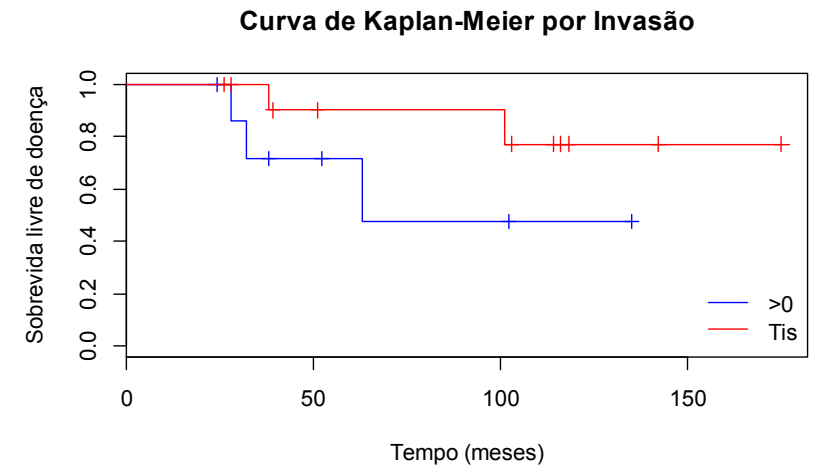

Figura 11 - Correlação entre sobrevida livre de doença e espessura tumoral

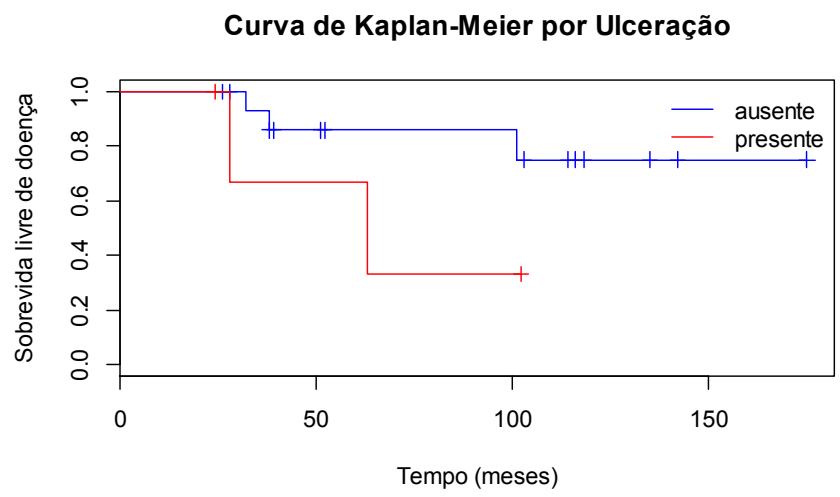

Figura 12 - Correlação entre sobrevida livre de doença e presença de ulceração à histopatologia

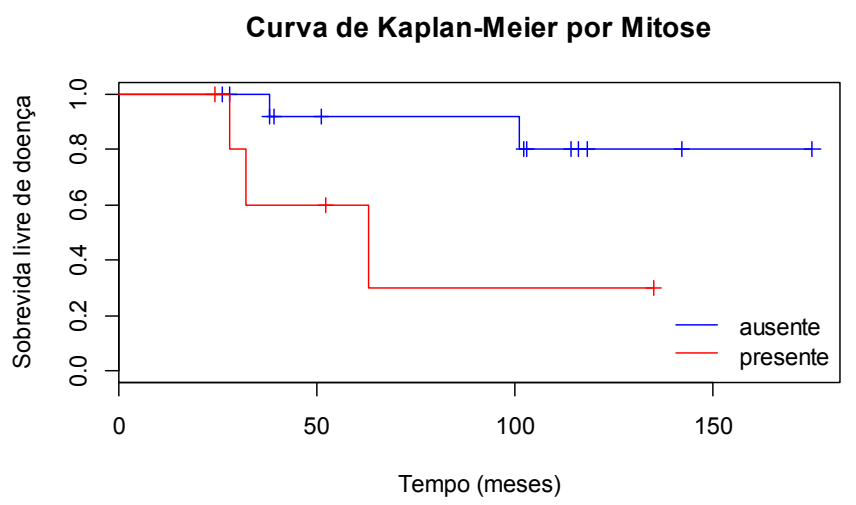

Figura 13 - Correlação entre sobrevida livre de doença e presença de mitose à histopatologia $(p=0,0511)$ 


\section{DISCUSSÃO}

\subsection{Aspectos Gerais das Características Clínicas e Sobrevida}

Assim como na literatura, observou-se discreta predominância na população feminina. Da mesma forma, a média de idade (52,14 anos) encontra-se na faixa mais acometida que abrange dos 50 aos 70 anos, havendo também casos diagnosticados na infância, como previsto na literatura. Por outro lado, nessa amostra, os pés foram mais atingidos do que as mãos, contradizendo o habitualmente observado nos outros trabalhos.

A sobrevida livre de doença é o período de tempo após o tratamento para uma doença específica, durante o qual o paciente sobrevive sem nenhum sinal de doença. ${ }^{137}$ Sem dúvida, é um parâmetro de avaliação do benefício clínico oriundo da terapia.

Não havendo protocolos de tratamento estabelecidos para o MU, sua abordagem evolui de acordo com as evidências. Observa-se, no entanto, escassez de dados que impede concluir sobre os resultados relacionados ao tratamento e, consequentemente, à sobrevida.

Segundo uma revisão, $81,7 \%$ (722 de 884) dos casos relatados na literatura não especificam a profundidade do melanoma na apresentação, sendo esse um dos fatores prognósticos mais importantes. A sobrevida é por vezes relatada nesses grupos de pacientes cujas informações são incompletas e dificultam as conclusões baseadas em evidências. ${ }^{138}$

De acordo com diferentes trabalhos, a sobrevida em 5 anos dos pacientes com melanoma ungueal varia entre 18 e 60,5\%. ${ }^{50,52,136}$ Nesse estudo, o seguimento mínimo foi estabelecido em 24 meses para maior aproveitamento da amostra, dado a raridade do tumor. Com base na curva de Kaplan-Meier global, a sobrevida em 2 anos pode ser estimada em 100\% e em 5 anos, aproximadamente $80 \%$. 
As variáveis como gênero, localização da lesão, espessura (índice de Breslow), ulceração e mitose não demonstraram impacto estatisticamente significante em relação à sobrevida. Tampouco a escolha da técnica cirúrgica demonstrou qualquer influência, uma vez que $p$ mostrou-se maior que 0,05. Porém, a variável mitose apresentou $p=0,0511$, valor próximo do nível de significância para influenciar em algum evento.

Esse resultado é corroborado por outros trabalhos da literatura, que demonstram não haver piora do prognóstico a despeito da abordagem cirúrgica. ${ }^{27,50,117,139} \mathrm{Em}$ contrapartida, a literatura demonstra que características histológicas, como espessura do tumor ${ }^{27,139}$, presença de mitose e ulceração, são prognósticos para MU. Além dessas, a localização (mãos versus pés) também é relatada como fator prognóstico. ${ }^{139}$ Provavelmente, o pequeno número de casos justifique a dificuldade de confirmar os dados da literatura.

A espessura tumoral, medida em milímetros pelo índice de Breslow, é utilizada para tumores invasivos, isto é, que ultrapassaram a epiderme. Os tumores finos possuem até $1 \mathrm{~mm}$, intermediários de $1 \mathrm{~mm}$ a $4 \mathrm{~mm}$ e os espessos são maiores que $4 \mathrm{~mm}$. Tumores in situ são não invasivos e, a rigor, não estão incluídos nessa classificação, uma vez que se restringem à epiderme.

Com base nesse conceito, nota-se que poucos trabalhos incluem casos de melanoma in situ de maneira explícita. Na maioria das vezes, descrevem casos com Breslow $<1 \mathrm{~mm}$, sem especificar os in situ, o que deixa a interpretação duvidosa, ou, de fato, não incluíram casos in situ. Há forte evidência de que a cirurgia funcional seja segura para os melanomas in situ e, mais do que isso, há tendência em utilizar a técnica para tumores cada vez mais espessos.

Dois dos nove casos in situ tratados com funcional neste estudo apresentaram recidiva local após 11 meses e 77 meses de seguimento. A primeira em paciente de 81 anos, novamente in situ e a margem foi ampliada. A segunda, em paciente de 42 anos se reapresentou como tumor invasivo $(0,6 \mathrm{~mm})$ e foi amputado. Ambos do tipo lentiginoso acral e sem recidiva até o momento. Talvez, fatores geneticamente determinados poderiam influenciar a resposta imune do paciente, justificando diferentes evoluções. 
A Tabela 4 apresenta os casos in situ da literatura tratados com cirurgia conservadora e as respectivas recidivas. Observa-se que o tempo de recidiva variou de 5 a 36 meses.

Tabela 4 - Casos in situ da literatura tratados com cirurgias conservadoras, e tempo em que ocorreram as respectivas recidivas

\begin{tabular}{|c|c|c|c|c|}
\hline Autor, ano & $\begin{array}{l}\text { Pacientes } \\
\text { (N) }\end{array}$ & $\begin{array}{l}\text { Número de } \\
\text { recidivas }\end{array}$ & $\begin{array}{c}\text { Tempo da } \\
\text { recidiva } \\
\text { (meses) }\end{array}$ & $\begin{array}{l}\text { Tempo de } \\
\text { seguimento } \\
\text { (meses) }\end{array}$ \\
\hline Banfield e cols. ${ }^{140}, 1999$ & 1 (Mohs) & - & - & 24 \\
\hline Clarkson e cols. ${ }^{26}, 2002$ & 1 & 0 & - & Não consta \\
\hline High e cols. ${ }^{12}, 2004$ & 4 & 1 & 5 & $10-29(24,3)$ \\
\hline Lazar e cols. ${ }^{132}, 2005$ & 9 & 0 & - & $6-84(50,4)$ \\
\hline Cohen e cols. ${ }^{141}, 2008$ & 6 & 1 & 18 & Não consta \\
\hline Imakado e cols. ${ }^{28}, 2008$ & 2 & 0 & - & $6-48$ \\
\hline Duarte e cols. ${ }^{29}, 2010$ & 1 & 0 & - & 12 \\
\hline Sureda e cols. ${ }^{1}, 2011$ & 5 & 0 & - & 24 a 84 \\
\hline Chow e cols. ${ }^{134}, 2013$ & 1 & 0 & - & Não consta \\
\hline Hayashi e cols. ${ }^{142}, 2012$ & 1 & 0 & - & 24 \\
\hline Debarbieux e cols. ${ }^{143}, 2012$ & 7 & 0 & - & Não consta \\
\hline Stolf e cols. ${ }^{144}, 2012$ & 1 & 0 & - & 24 \\
\hline Haddock e cols. ${ }^{145}, 2014$ & 9 & 0 & - & $\begin{array}{c}20-103 \\
(50,7)\end{array}$ \\
\hline Nakamura e cols. ${ }^{146}, 2015$ & 48 & 4 & $14,18,31,36$ & $\begin{array}{c}24-209 \\
(57,6)\end{array}$ \\
\hline Haneke e cols. ${ }^{147} 2016$ & 1 & 0 & - & 5 \\
\hline
\end{tabular}

Da mesma forma, a evolução dos tumores invasivos também pode ser divergente. Um paciente (59 anos) com tumor de $2,5 \mathrm{~mm}$, submetido à funcional por não consentir a amputação, apresentou metástase linfonodal após 18 meses. Paralelamente, outro paciente (49 anos) com tumor de 3,5 $\mathrm{mm}$, tratado com funcional pelo mesmo motivo, está há mais de 11 anos em seguimento sem sinais de recidiva. Ambos do mesmo tipo histológico, lentiginoso acral.

Dois pacientes com tumor invasivo abordados com amputação apresentaram metástase a distância. Um com 2,65 mm de profundidade apresentou metástase pulmonar após 26 meses, com óbito após 2 meses. 0 
outro, com Breslow de 1,9 mm, desenvolveu metástase mediastinal e em tronco celíaco após 24 meses. Fez quimioterapia e imunoterapia, apresentando-se sem recidiva. É interessante destacar que ambos os casos apresentavam o mesmo tipo histológico (lentiginoso acral), presença de ulceração e mitose à histopatologia. Por outro lado, houve caso de $12 \mathrm{~mm}$ de espessura, do tipo lentiginoso acral, com presença de úlcera e mitose à histopatologia há 2 anos em seguimento sem sinais de recidiva.

Ainda que a amostra se mostre bastante homogênea quanto às características analisadas, mais estudos serão necessários para identificar os fatores determinantes de evoluções díspares. Ao analisar os casos em separado, reafirma-se o apresentado pela análise estatística quanto a não interferência da espessura tumoral ou da cirurgia escolhida na sobrevida.

Outro fator que contribui para a homogeneidade da amostra é o fato de apenas dois cirurgiões terem tratado dos pacientes, o que reduz possíveis vieses de técnica. $O$ estudo também se destaca por incluir ambas as técnicas (funcional e amputação) para o tratamento de casos com tumores in situ e invasivos. Dos trabalhos comparativos com ambas as técnicas, este é o maior deles em número de casos de $\mathrm{MU}$ in situ.

Nesta pesquisa, a cirurgia funcional quando comparada à amputação não se correlaciona com um melhor prognóstico, mesmo em pacientes que apresentam Breslow maior. Da mesma forma, a amputação não demonstrou aumentar a sobrevida. A única variável que chegou mais próximo de alterar a sobrevida foi a presença de mitose à histopatologia.

O número de casos certamente é um limitante das análises, porém, tratando-se de um tumor raro, observa-se a mesma dificuldade em outros trabalhos. Estudos com maior número de casos, talvez multicêntricos e com o maior número de informações possíveis, são necessários para conclusões definitivas. 


\section{CONCLUSÃO}

Não houve alteração da sobrevida dos pacientes com MU tratados com amputação ou cirurgia funcional.

As características histopatológicas, como nível de invasão, presença de mitose e ulceração ao histopatológico não influenciaram a sobrevida. 


\section{REFERÊNCIAS}

1. Sureda N, Phan A, Poulalhon N, Balme B, Dalle S, Thomas L. Conservative surgical management of subungual (matrix derived) melanoma: report of seven cases and literature review. $\mathrm{Br}$ Association of Dermatol. 2011;165:852-858.

2. Haneke E. Ungual melanoma - controversies in diagnosis and treatment. Dermatologic Therapy. 2012:(25):510-524.

3. Kato T, Suetake T, Sugiyama Y, Tabata N, Tagami H. Epidemiology and prognosis of subungual melanoma in 34 Japanese patients. $\mathrm{Br} \mathrm{J}$ Dermatol. 1996;134:383-387.

4. Thai KE, Young R, Sinclair RD. Nail apparatus melanoma. Australas $J$ Dermatol. 2001;42:71-81.

5. Tosti A, Piraccini BM, Cagalli AE. In situ melanoma of the nail unit in children: report of 2 cases in Caucasian fair skinned children. PediatrDermatol. 2012;29:79-83.

6. Quinn MJ, Thompson JE, Crotty K, et al. Subungual melanoma of the hand. J Hand Surg Am. 1996;21:506-11.

7. Seui M, Takematsu H, Hosokawa M, et al. Acral melanoma in Japan. J Invest Dermatol. 1983:80(1 Suppl.):56s-60s.

8. Phan A, Touzet S, Dalle S, Ronger-Savlé S, Balme B, Thomas L. Acrallentiginous melanoma: a clinic prognostic study of 126 cases. $\mathrm{Br} J$ Dermatol. 2006;155:561-569.

9. Braun RP, Baran R, Le Gal FA et al. Diagnosis and management of nail pigmentations. J Am Acad Dermatol. 2007;56:835-47.

10. Kovich OI, Soldano AC. Clinical pathologic correlations for diagnosis and treatment of nail disorders. Dermatol Ther. 2007;20:11-16.

11. Thomas L, Dalle S. Dermoscopy provides useful information for the management of melanonychiastriata. Dermatol Ther. 2007;20:3-10. 
12. High WA, Quirey RA, Guillén DR, et al: Presentation, histopathologic findings, and clinical outcomes in 7 cases of melanoma in situ of the nail unit. Arch Dermatol. 2004;140:1102-1106.

13. Baran R, Barth J, Dawber R: Periungual tissue disorders; in Baran R, Barth J, Dawber R (eds): Nail Disorders: Common Presenting Signs, Differential Diagnosis and Treatment. Dunitz. 1991:123-126.

14. Haneke E, Binder D. Subungual melanoma with linear nail pigmentation. Hautarzt. 1978 Jul;29(7):389-91.

15. Gray RJ, Pockaj BA, Vega ML et al. Diagnosis and treatment of malignant melanoma of the foot. Foot Ankle Int. 2006;27:696-705.

16.Wagner A, Garrido I, Ferron $G$ et al. Subungual melanoma: for a conservative approach on the thumb scale. Ann Plast Surg. 2007;59:344-8.

17. Eedy DJ: Surgical treatment of melanoma. Br J Dermatol. 2003;149:2-12.

18. Baran R, Haneke E, Drapé J-L, Zook EG, Kreusch JF. Tumours of the nail apparatus and adjacent tissues. In: Baran R, et al., eds. Baran and Dawber's Diseases of the Nails and their Management. 3rd ed. Oxford: Blackwell; 2001. p.515-630.

19. Elpern D. Subungual melanoma vs. hematoma. Virtual Grand Rounds Dermatology [Internet]. 2013 Jun 16 [cited 2011 Jan 12]. Available from: http://www.vgrd.org/archive/cases/2001/submel/submel.html.

20. Haneke E, Baran R. Subungual Tumours. Z Hautkr. 1982;57:355-362.

21. Wallberg B, Hansson J. Delayed diagnosis of subungual melanoma. Two cases were misjudged as onychomycosis. Läkartidningen. 1997;94:25432544.

22. Braham C, Fraiture AL, Quatresooz P, Piérard-Franchimont C, Piérard GE. "Banal dermatomycoses" that cannot be overlooked. Rev Med Liège. 2002;57:317-319.

23. Soon SL, Solomon AR Jr, Papadopoulos D, Murray DR, McAlpine B, Washington $\mathrm{CV}$. Acrallentiginous melanoma mimicking benign disease: the Emory experience. J Am Acad Dermatol. 2003;48:183-188. 
24. Haneke E. Surgical therapy of acral and subungual melanomas. In: Rompel R, Petres J, eds. (Hrsg). Operational and oncological dermatology. Advances in surgical and oncological dermatology. Berlin: Springer. 1999;15:210-214.

25. Moncrieff MD, Thompson JF, Quinn MJ et al. Reconstruction after wide excision of primary cutaneous melanomas: part II - the extremities. Lancet Oncol. 2009;10:810-15.

26. Clarkson JH, McAllister RM, Cliff SH et al. Subungual melanoma in situ: two independent streaks in one nail bed. Br J PlastSurg. 2002;55:165-7.

27. Moehrle M, Metzger S, Schippert W, Garbe C, Rassner G, Breuninger H. "Functional" surgery in subungual melanoma. Dermatol Surg. 2003;29:366374.

28. Imakado S, Sato H, Hamada K. Two cases of subungual melanoma in situ. J Dermatol. 2008;35:754-8.

29. Duarte AF, Correia O, Barros AM et al. Nail matrix melanoma in situ: conservative surgical management. Dermatology. 2010;220:173-5.

30. Smock ED, Barabas AG, Geh JL. Reconstruction of a thumb defect with Integra following wide local excision of a subungualmelanoma. J Plast Reconstr Aesthet Surg. 2010;63:e36-7.

31. Skin cancers - How common is skin cancer? World Health Organization. [Internet]. 2016. [3 screens]. Available from: http://www.who.int/uv/faq/skincancer/en/index1.html.

32. Tipos de câncer - Pele melanoma. Instituto Nacional do Câncer José Alencar Gomes da Silva - Inca. [Internet]. 2016. [1 tela]. Disponível em: http://www2.inca.gov.br/wps/wcm/connect/tiposdecancer/site/home/pele_m elanoma.

33. American Cancer Society. Cancer Facts \& Figures 2008. Atlanta: American Cancer Society; 2008. Available from: http://www.cancer.org/acs/groups/content/@nho/documents/document/200 8cafffinalsecuredpdf.pdf. 
34. Garbe C: Risk factors for the development of malignant melanoma and identification of risk groups in German-speaking regions. Hautarzt. 1995;46:309-314.

35. Surveillance, Epidemiology and End Results (SEER) Program. National Cancer Institute, DCCPS, Surveillance Research Program, Cancer Statistics Branch; Apr. 2008. SEER ${ }^{\star}$ Stat Database. Available from: www.seer.cancer.gov .

36. Markovic, SN, Erickson, LA, Rao, RD et al, Melanoma Study Group of the Mayo Clinic Cancer Center. Malignant melanoma in the 21st century, part 1: epidemiology, risk factors, screening, prevention, and diagnosis. Mayo Clin Proc. 2007;82:364-380.

37. Bastian BC, Kashani-Sabet M, Hamm H, et al. Gene amplifications characterize acral melanoma and permit the detection of occult tumor cells in the surrounding skin. Cancer Res. 2000;60:1968-1973.

38. Curtin JA, Fridlyand J, Kageshita T, et al. Distinct sets of genetic alterations in melanoma. N Engl J Med. 2005;353:2135-2147.

39. Bauer J, Bastian BC. Distinguishing melanocytic nevi from melanoma by DNA copy number changes: comparative genomic hybridization as a research and diagnostic tool. Dermatol Ther. 2006;19:40-49.

40. North JP, Kageshita T, Pinkel D, LeBoit PE, Bastian BC. Distribution and significance of occult intraepidermal tumor cells surrounding primary melanoma. J Invest Dermatol. 2008;128:2024-2030.

41. Viros A, Fridlyand J, Bauer J, et al. Improving melanoma classification by integrating genetic and morphologic features. PLoS Med. 2008;5(6):e120.

42. Fargnoli MC, Pike K, Pfeiffer RM, et al. MC1R variants increase risk of melanomas harboring BRAF mutations. J Invest Dermatol. 2008;128:24852490.

43. Gerami P, Jewell SS, Morrison LE, et al. Fluorescence in situ hybridization (FISH) as an ancillary diagnostic tool in the diagnosis of melanoma. Am J Surg Pathol. 2009;33:1146-1156.

44. Koller KM, Wang W, Schell TD et al. Malignant melanoma-The cradle of anti-neoplastic immunotherapy. Crit Rev Oncol Hematol. 2016;106:25-54. 
45. Boyer A. Fungus nematode of little fingers. Gaz Med Paris. 1834;2:212.

46. Hutchinson J. Melanosis often not black: melanotic whitlow. $\mathrm{Br}$ Med J. 1886;1:491.

47. Kottschade LA, Grotz TE, Dronca RS, et al Salomao DR, Pulido JS, Wasif $\mathrm{N}$, Jakub JW, Bagaria SP, Kumar R, Kaur JS, Morita SY, Moran SL, Nguyen JT, Nguyen EC, Hand JL, Erickson LA, Brewer JD, Baum CL, Miller RC, Swanson DL, Lowe V, Markovic SN. Rare presentations of primary melanoma and special populations: a systematic review. Am J Clin Oncol. 2014 Dec;37(6):635-41.

48. Berker DAR, Baran R. Disorder of Nails. In: Burns T, Breathnach S, Cox N, Griffiths C, editors. Rook's Textbook of Dermatology. Oxford: Blackwell Publishers; 2010. Chap.65, p 1-57.

49. Levit EK, Kagen $\mathrm{MH}$, Scher RK, et al. The $A B C$ rule for clinical detection of subungual melanoma. J Am Acad Dermatol. 2000;42:269-74.

50. Nguyen, JT.; Bakri, K.; Nguyen, EC., et al. Surgical management of subungual melanoma: a single institution experience of 124 cases. Ann Plast Surg. 2013;71(4):346-54.

51. Tan KB, Moncrieff M, Thompson JF, et al. Subungual melanoma: a study of 124 cases highlighting features of early lesions, potential pitfalls in diagnosis, and guidelines for histologic reporting. Am J Surg Pathol. 2007;31:1902-12.

52. O'Leary JA, Berend KR, Johnson JL, et al. Subungual melanoma. A review of 93 cases with identification of prognostic variables. Clin Orthop. 2000;378:206-212.

53. Banfield CC, Redburn JC, Dawber RP. The incidence and prognosis of nail apparatus melanoma. A retrospective study of 105 patients in four English regions. Br J Dermatol. 1998;139:276-9.

54. Miura S, Jimbow K. Clinical characteristics of subungual melanomas in Japan: case report and a questionnaire survey of 108 cases. $J$ Dermatol. 1985;12:393-402.

55. Blessing K, Kernohan NM, Park KG. Subungual malignant melanoma: clinicopathological features of 100 cases. Histopathology. 1991;19:425-9. 
56. Mohrle M, Hafner HM. Is subungual melanoma related to trauma? Dermatology. 2002;204:259-61.

57. Bruls WA, Slaper H, van der Leun JC, Berrens L: Transmission of human epidermis and stratum corneum as a function of thickness in the ultraviolet and visible wavelengths. Photochem Photobiol. 1984;40:485-494.

58. Stern DK, Creasey AA, Quijije J, Lebwohl MG. UV-A and UV-B penetration of normal human cadaveric fingernail plate. Arch Dermatol. 2011;147:439441.

59. Haneke E, Baran R. Longitudinal melanonychia. Dermatol Surg. 2001;27:580-584.

60. Jin H, Kim JM, Kim GW, Song M, Kim HS, Ko HC, Kim BS, Kim MB. Diagnostic criteria for and clinical review of melanonychia in Korean patients. J Am Acad Dermatol. 2016;30:1-7.

61. Kopf AW. Hutchinson's sign in subungual melanoma. Am J Dermatopathol. $1981 ; 3: 210-2$.

62. Hutchinson J. Melanosis often not black; melanotic whitlow. $\mathrm{Br}$ Med $\mathrm{J}$. 1886;1:491.

63. Baran R, Kechijian P. Hutchinson's sign: a reappraisal. J Am Acad Dermatol. 1996;34(1):87-90.

64. Clark $\mathrm{WH}$, Bemadino EA, Reed RJ, et al. Acral lentiginous melanomas including melanomas of mucous membranes. In: Clark WH, Goldman LI, Mastrax E, eds. Human malignant melanoma. New York: Grune \& Stratton; 1979. p.109-24.

65. Gibson SH, Montgomery H, Wollner B, et al. Melanotic whitlow (subungual melanoma). J Invest Dermatol. 1957;29:119-29.

66. Patterson RH, Helwig EB. Subungual melanoma: a clinical pathologic study. Cancer. 1980;46:2074-87.

67. Takematsu H, Obata M, Tomita $Y$, et al. Subungual melanoma: a clinicopathological study of 16 Japanese cases. Cancer. 1985;55:2725-31.

68. Shukla VK, Hughes LE. Differential diagnosis of subungual melanoma from a surgical point of view. Br J Surg. 1989;76:1156-1160. 
69. Di Chiacchio N, Hirata SH, Enokihara MY, Michalany NS, Fabbrocini G, Tosti A. Dermatologists' accuracy in early diagnosis of melanoma of the nail matrix. Arch Dermatol. 2010;146(4):382-7.

70. Baran R, Kechijian P. Longitudinal melanonychia (melanonychia striata): diagnosis and management. J Am Acad Dermatol. 1989;21:1165-1175.

71. Lateur N, Andre J. Melanonychia diagnosis and treatment. Dermatol Ther. 2002;15:131-141.

72. Jellinek N. Nail matrix biopsy of longitudinal melanonychia: diagnostic algorithm including the matrix shave biopsy. J Am Acad Dermatol. 2007;56(5):803-810.

73. Tosti A, Piraccini BM, Cadore de Farias D. Dealing with melanonychia. Semin Cutan Med Surg. 2009;28(1):49-54.

74. Finley RK 3rd, Driscoll DL, Blumenson LE, Karakousis CP. Subungual melanoma: an eighteen-year review. Surgery. 1994;116:96-100.

75. Vrouenraets BC,Kroon BB,KlaaseJM,Von Geel BN,Eggermont AM,Mooi WJ,et al.Regional isolated perfusion with melphalan for patients with subungual melanoma. Eur J Surg Oncol. 1993;19:37-42.

76. Orlow S. Melanomas in children. Pediatr Rev. 1995;16:365-9.

77. Rigby HS, Briggs JC. Subungual melanoma: a clinico-pathological study of 24 cases. Br J Plast Surg. 1992;45:275-278.

78. O'Toole E, Stephens R, Young M, Tanner A, Barnes L. Subungual melanoma: a relation to direct injury? J Am Acad Dermatol. 1995;33:525-8.

79. Scher RK, Silvers DN. Longitudinal melanonychia striata. J Am Acad Dermatol. 1991;24:1035.

80. Tom DW, Scher RK. Biopsies of nails. Melanonychia striata in longitudinem. Am J Dermatopathol. 1985;7(Suppl):161-3.

81. Saida T, Oshima Y. Clinical and histopathological characteristics of the early lesions of subungual melanoma. Cancer. 1989;63:556-60.

82. Black W, Wiggins C. Melanoma among southwestern Indians. Cancer. 
1985;55:2899-902.

83. Molina D, Sanchez J. Pigmented longitudinal bands of the nail. Am J Dermatopathol. 1995;17:539-41.

84. Kato T, Usuba $\mathrm{Y}$, Takematsu H, Kumasaka N, Tanita $\mathrm{Y}$, Hashimoto $\mathrm{K}$, et al. A rapidly growing pigmented nail streak in diffuse melanosis of the nail. Cancer 1989;64:2191-7.

85. Ackerman AB. Subtle clues to diagnosis by conventional microscopy: signs that stamp pigmented melanotic nevi as benign. Am J Dermatopathol. 1982;4:461-6.

86. Beltrani V, Scher RK. Evaluation and management of melanonychia striata in a patient receiving phototherapy. Arch Dermatol. 1991;12:319-20.

87. Glat PM, Spector JA, Roses DF, Shapiro RA, Harris MN, Bealey RW, Grossman JA. The management of pigmented lesions of nail bed. Ann Plast Surg. 1996; 37(2):125-34.

88. Bibbo C, Brolin R, Warren A, Franklin I. Current therapy for sub-ungual melanoma. J Foot Ankle Surg. 1994;33:184-93.

89. Papachristou D, Fortner J. Melanoma arising under the nail. J Surg Oncol. 1982;21:219-22.

90. Krige JE, Hudson DA, Johnson CA, King HS, Chetty R. Subungual melanoma. South Afr J Surg. 1995;33:10-4.

91. Kelly J, Yeatman J, Regalia C, Mason G, Henham A. A high incidence of melanoma found in patients with multiple dysplastic naevi by photographic surveillance. Med J Aust. 1997;167:191-4.

92. Argenziano G, Soyer HP. Dermoscopy of pigmented skin lesions - a valuable tool for early diagnosis of melanoma. Lancet Oncol. 2001;2:443-9.

93. Bafounta ML, Beauchet A, Aegerter P, Saiag P. Is dermoscopy (epiluminescence microscopy) useful for the diagnosis of melanoma? Results of a meta-analysis using techniques adapted to the evaluation of diagnostic tests. Arch Dermatol. 2001;137(10):1343-50. 
94. Ronger S, Touzet S, Ligeron C, Balme B, Viallar AM, Barrut D, Colin C, Thomas L. Dermoscopic examination of nail pigmentation. Arch Dermatol. 2002;138(10):1327-33.

95. Tosti A, Argenziano G. Dermoscopy allows better management of nail pigmentation. Arch Dermatol. 2002;138:1369-70.

96. Braun RP, Rabinovitz HS, Oliviero M, Kopf AW, Saurat JH. Dermoscopy of pigmented skin lesions. J Am Acad Dermatol. 2005;52:109-21.

97. Hirata SH, Yamada S, Almeida FA, Enokihara MY, Rosa IP, Enokihara MM, Michalany NS. Dermoscopic examination of the nail bed and matrix. Int $J$ Dermatol. 2006;45(1):28-30.

98. Menzies SW. Cutaneous melanoma: making a clinical diagnosis, present and future. Dermatol Ther. 2006;19:32-9.

99. Di Chiacchio N, Loureiro WR, Michalany NS, Kezam Gabriel FV. Tangential Biopsy Thickness versus Lesion Depth in Longitudinal Melanonychia: A Pilot Study. Dermatol Res Pract. 2012;2012:353864.

100. Astur Mde M, Farkas CB, Junqueira JP, Enokihara MM, Enokihara MY, Michalany N, Hirata SH. Reassessing Melanonychia Striata in Phototypes IV, V, and VI Patients. Dermatol Surg. 2016 Feb;42(2):183-90.

101. Di Chiacchio N, Farias DC, Piraccini BM, Hirata SH, Richert B, Zaiac M, Daniel R, Fanti PA, Andre J, Ruben BS, Fleckman P, Rich P, Haneke E, Chang P, Cherit JD, Scher R, Tosti A. Consensus on melanonychia nail plate dermoscopy. An Bras Dermatol. 2013;88(2):313-7.

102. Phan A1, Dalle S, Touzet S, Ronger-Savlé S, Balme B, Thomas L. Dermoscopic features of acral lentiginous melanoma in a large series of 110 cases in a white population. Br J Dermatol. 2010;162(4):765-71.

103. Hirata SH, Yamada S, Enokihara MY, et al. Patterns of nail matrix and bed of longitudinal melanonychia by intraoperative dermatoscopy. J Am Acad Dermatol. 2011;65(2):297-303.

104. Hirata SH, Yamada S, Almeida FA, Tomomori-Yamashita J, Enokihara MY, Paschoal FM, et al. Dermoscopy of the nail bed and matrix to assess melanonychia striata. J Am Acad Dermatol. 2005;53:884-6.

105. Iorizzo M, Tosti A, Di Chiacchio N, Hirata SH, Misciali C, Michalany N, et 
al. Nail melanoma in children: differential diagnosis and management. Dermatol Surg. 2008;34:974-8.

106. Ruben BS. Pigmented lesions of the nail unit. Semin Cutan Med Surg. 2015 Jun;34(2):101-8

107. Clark, W. H., Jr., Elder, D. E., and Van Horn, M. The biologic forms of malignant melanoma. Hum Pathol. 1986;17:443-450.

108. Mendonça IRSM, Kac BK, Silva RT, et al. Melanoma do aparelho ungueal. An Bras Dermatol. 2004;79(5):575-580.

109. Shin HT, Jang KT, Mun GH, Lee DY, Lee JB. Histopathological analysis of the progression pattern of subungual melanoma: late tendency of dermal invasion in the nail matrix area. Mod Pathol. 2014 Nov;27(11):1461-7.

110. Feibleman CE, Stoll H, Maize JC. Melanomas of the palm, sole, and nailbed: a clinicopathologic study. Cancer. 1980;46:2492-2504.

111. Elpern D. Subungual melanoma vs. hematoma. Virtual Grand Rounds Dermatology [Internet] June 16th, 2001. [cited 2011 Jan 12].Available from: http://www.vgrd.org/archive/cases/2001/submel/submel.html.

112. Wallberg B, Hansson J. Delayed diagnosis of subungual melanoma. Two cases were misjudged as onychomycosis [In Swedish]. Läkartidningen. 1997;94:2543-2544.

113. Soon SL, Solomon AR Jr, Papadopoulos D, Murray DR, McAlpine B, Washington $\mathrm{CV}$. Acral lentiginous melanoma mimicking benign disease: the Emory experience. J Am Acad Dermatol. 2003;48:183-188.

114. Bradford PT, Goldstein AM, McMaster ML, Tucker MA. Acral lentiginous melanoma: incidence and survival patterns in the United States, 19862005. Arch Dermatol. 2009;145:427-434.

115. Metzger S, Ellwanger U, Stroebel W, Schiebel U, Rassner G, Fierlbeck G. Extent and consequences of physician delay in the diagnosis of acral melanoma. Melanoma Res. 1998;8:181-186.

116. Slingluff CL Jr, Vollmer R, Seigler HF. Acral melanoma: a review of 185 patients with identification of prognostic variables. J Surg Oncol. 1990; 45:91-98. 
117. Park KG, Blessing K, Kernohan NM. Surgical aspects of subungual malignant melanoma. The Scottish melanoma group. Ann Surg. 1992; 216:692-695.

118. Orfanos CE, Jung EG, Rasser G, Wolff HH, Garbe C. Position and recommendations of the Malignant Melanoma Committee of the German Society of Dermatology on diagnosis, treatment and after-care of malignant melanoma of the skin. Status 1993/94. Hautarzt. 1994;45: 284-291.

119. Haneke E. Matrix surgery: tangential excision. In: Richert B, Di Chiacchio $\mathrm{N}$, Haneke E, eds. Nail surgery. London and New York: Informa Health Care; 2011. p. 129-131.

120. Pack GT, Oropeza R. Subungual melanoma. Surg Gynecol Obstet. 1967;124:571-82.

121. Lingam MK, McKay AJ, Mackie RM, Aitchison T. Single-centre prospective study of isolated limb perfusion with melphalan in the treatment of subungual malignant melanoma. Br J Surg. 1995;82:1343-5.

122. Ross MI, Reintgen D, Balch CM. Selective lymphadenectomy: emerging role for lymphatic mapping and sentinel node biopsy in the management of early stage melanoma. Semin Surg Oncol. 1993;9:219-23.

123. Wagner JD, Gordon MS, Chuang TY, Coleman JJ. Current therapy of cutaneous melanoma. Plast Reconstr Surg. 2000;105:1774-99. 121.

124. Daly JM, Berlin R, Urmacher C. Subungual melanoma: a 25-year review of cases. J Surg Oncol. 1987;35:107-12.

125. Das Gupta T, Bradfield R. Subungual melanoma: 25-year review of cases. Ann Surg. 1965;161:545-552.

126. Heaton KM, el-Naggar A, Ensign LG, Ross MI, Balch CM. Surgical management and prognostic factors in patients with subungual melanoma. Ann Surg. 1994 Feb;219(2):197-204.

127. Balch CM, Gershenwald JE, Soong SJ, et al. Final version of 2009 AJCC melanoma staging and classification. J Clin Oncol. 2009;27:6199-6206.

128. Fortin PT, Freiberg AA, Rees R, et al. Malignant melanoma of the foot 
and ankle. J Bone Joint Surg Am. 1995;77:1396-1403.

129. Muchmore JH, Krementz ET, Carter RD, et al. Regional perfusion for the treatment of subungual melanoma. Am Surg. 1990;56:114-118.

130. Rayatt SS, Dancey AL, Davison PM. Thumb subungual melanoma: Is amputation necessary? J Plast Reconstr Aesthet Surg. 2007;60:635638.

131. Balch CM, Buzaid AC, Soong SJ, et al. Final version of the American Joint Committee on cancer staging system for cutaneous melanoma. $J$ Clin Oncol. 2001;19:3635-48.

132. Lazar A, Abimelec $P$, Dumontier $C$. Full thickness skin graft for nail unit reconstruction. J Hand Surg Br. 2005;30:194-198.

133. Nakamura $Y$, Fujisawa $Y$, Teramoto $Y$, Sato S, Yamada K, Sekine K, Fujimoto M, Otsuka F, Yamamoto A. Tumor-to-bone distance of invasive subungual melanoma: an analysis of 30 cases. J Dermatol. 2014 Oct;41(10):872-7.

134. Chow WT, Bhat W, Magdub S, Orlando A. In situ subungual melanoma: digit salvaging clearance. J Plast Reconstr Aesthet Surg. 2013;66(2):274-6.

135. Maekawa $T$, Komine M, Murata $S$, Ohtsuki M. Surgical treatment of subungual melanoma: a case treated with bone splitting of the distal phalanx. J Dermatol. 2014;41(10):944-6.

136. Bormann G, Marsch WC, Haerting J, Helm- bold P: Concomitant traumas influence prognosis in melanomas of the nail apparatus. $\mathrm{Br} J$ Dermatol. 2006;155:76-80.

137. $\mathrm{NCl}$, National Cancer Institute. Disease-free survival. Dictionary of Cancer Terms. 2010.2 Available from: http://www.cancer.gov/dictionary?CdrlD=44023.

138. Cochran AM, Buchanan PJ, Bueno RA Jr, Neumeister MW: Subungual melanoma: a review of current treatment. Plast Reconstr Surg. 2014;134:259-273.

139. Dika E, Patrizi A, Fanti PA, Chessa MA, Reggiani C, Barisani A, Piraccini BM. The Prognosis of Nail Apparatus Melanoma: 20 Years of Experience 
from a Single Institute. Dermatology. 2016;232(2):177-84.

140. Banfield CC1, Dawber RP, Walker NP, Stables GI, Zeina B, Schomberg $\mathrm{K}$. Mohs micrographic surgery for the treatment of in situ nail apparatus melanoma: a case report. J Am Acad Dermatol. 1999 Jan;40(1):98-9.

141. Cohen T, Busam K, Patel A, et al. Subungual melanoma: Management considerations. Am J Surg. 2008;195:244-248.

142. Hayashi K, Uhara H, Koga H, Okuyama R, Saida T. Surgical treatment of nail apparatus melanoma in situ: the use of Artificial dermis in reconstruction. Dermatol Surg. 2012;38(4):692-694.

143. Debarbieux S, Hospod V, Depaepe L, Balme B, Poulalhon N, Thomas L. Perioperative confocal microscopy of the nail matrix in the management of in situ or minimally invasive subungual melanomas. $\mathrm{Br} J$ Dermatol. 2012;167(4):828-836.

144. Stolf $\mathrm{H} \mathrm{O}$, Miot $\mathrm{H}$ A, Reis N A. Melanoma subungueal in situ tratado com cirurgia funcional. Diagn Tratamento. 2012;17(1):14-7.

145. Haddock NT, Wilson SC, Shapiro RL, Choi M. Wide local en bloc excision of subungual melanoma in situ, maximizing functional and aesthetic outcome: a retrospective review. Ann Plast Surg. 2014;73:640644.

146. Nakamura Y, Ohara K, Kishi A, Teramoto Y, Sato S, Fujisawa Y, et al. Effects of non-amputative wide local excision on the local control and prognosis of in situ and invasive subungual melanoma. Journal of Dermatology. 2015;42:861-866

147. Haneke E, Nakamura RC de; Papaiordanou F, Machado EA; D'Almeida LF. Cirurgia conservadora em caso de melanoma subungueal in situ. Surg Cosmet Dermatol. 2016;78(41):70-2. 